\title{
Algunos consejos útiles para el análisis cromatográfico de compuestos orgánicos volátiles
}

\author{
Elena Stashenko*, Jairo René Martínez \\ Centro de Investigación de Excelencia, CENIVAM, \\ Centro de Cromatografía y Espectrometría de Masas, \\ Escuela de Química, Universidad Industrial de Santander, \\ UIS, Bucaramanga, Colombia \\ e-mail:elena@tucan.uis.edu.co
}

\section{Resumen}

El análisis cromatográfico confiable de mezclas de compuestos orgánicos volátiles es el resultado de la atención cuidadosa a detalles del proceso de preparación de la muestra y de la selección del sistema cromatográfico a emplear y sus condiciones de operación. En este artículo se discuten los efectos de las principales variables que inciden en el análisis cromatográfico de compuestos orgánicos volátiles y se presentan algunas recomendaciones para varios procedimientos, junto con varios ejemplos que ilustran su aplicación.

\section{Palabras clave}

Cromatografía de gases; cromatografía de gases acoplada a espectrometría de masas; compuestos orgánicos volátiles.

\section{Some useful tips for the chromatographic analysis of volatile organic compounds}

\section{Abstract}

The reliable chromatographic analysis of mixtures of volatile organic compounds is the result of careful attention to details during sample preparation, chromatographic system and operating conditions selection. This article discusses the effects of the main variables that have incidence on the chromatographic analysis of volatile organic compounds. Recommendations are presented for various procedures, together with various examples that illustrate their application.

Keywords

Gas chromatography; gas chromatography-mass spectrometry; volatile organic compounds. 


\section{Introducción}

Las ventajas comparativas de la técnica de cromatografía de gases acoplada a espectrometría de masas (GC-MS) se destacan fundamentalmente en el campo del análisis de compuestos volátiles. En términos generales, los compuestos volátiles son aquellas moléculas que poseen presión de vapor alta (temperaturas de ebullición relativamente bajas, generalmente, menores de $200-250{ }^{\circ} \mathrm{C}$ ) y peso molecular que no supera 250-300 Da; son sustancias que pueden tener diversos grupos funcionales, e.g., grupos aldehído, alcohol, amino, sulfuro, entre otros, de polaridad media, algunos, parcialmente o muy solubles en agua. Muchos de estos compuestos poseen olor y, varios, umbrales de percepción odorífera muy bajos; por ejemplo, compuestos azufrados (diversos sulfuros presentes en ajo, cebolla, huevo podrido, etc.). El análisis de sustancias volátiles es importante y se requiere en diferentes ramas del saber químico: 1) Ambiental (BTEX, VOC, THM, hidrocarburos volátiles, etc.); 2) Productos naturales (metabolitos secundarios volátiles, e.g., aceites esenciales, fragancias florales, aleloquímicos, feromonas, etc.); 3) Control de calidad de productos finales (per- fumes, productos de aseo personal, solventes residuales en fármacos, compuestos de bajo peso molecular en polímeros, entre ellos, monómeros, plastificantes y otros aditivos; 4) Alimentos (aromatizantes, saborizantes, compuestos off-flavor, etc.); 5) Estudios toxicológicos y forenses (residuos de explosivos y derivados, acelerantes de incendios maliciosos o premeditados, gases venenosos, compuestos tóxicos, solventes y metabolitos de drogas en fluidos fisiológicos, etc.) y 6) Investigaciones bio-médicas (compuestos biogénicos, e.g., volátiles en aliento, etc.), entre algunos otros campos. En la Tabla 1 aparecen algunas referencias sobre el análisis por GC-MS de compuestos volátiles, según el campo de aplicación.

El análisis de compuestos orgánicos volátiles (VOC) posee sus particularidades; entre algunas, figuran las siguientes: 1) Son mezclas complejas, multicomponente, que pueden contener más de 300 compuestos; por ejemplo, en el aliento humano se han registrado centenares de moléculas diferentes ${ }^{[38]}$; las fragancias florales pueden tener hasta mil sustancias de diversa naturaleza química ${ }^{[39]}$; en el aroma de café, según su procedencia, el grado y la duración de tostado del grano, el tipo de café (C. arabica, C. robusta, híbridos o variedades) se han encontrado más

Tabla 1 Algunas aplicaciones de la técnica GC-MS para el estudio de compuestos orgánicos volátiles.

\begin{tabular}{|c|c|}
\hline Área de aplicación & Objetos de análisis \\
\hline Ambiental & Contaminantes volátiles: BTEX, VOC, trihalometanos ${ }^{[1-3]}$, hidrocarburos volátiles \\
\hline Productos naturales & $\begin{array}{l}\text { Metabolitos secundarios volátiles de plantas: aceites esenciales }{ }^{[4]} \text {, fragancias } \\
\text { florales }^{[5-9]} \text { aleloquímicos, feromonas }{ }^{[10,11]}\end{array}$ \\
\hline Ciencias forenses & $\begin{array}{l}\text { Acelerantes de incendio }{ }^{[12-14]} \text {, residuos de explosivos }{ }^{[15,16]} \text {, compuestos volátiles } \\
\text { en muestras de evidencias forenses } \\
{[17,18]}\end{array}$ \\
\hline Estudios biomédicos & $\begin{array}{l}\text { Muestras biológicas: aliento }{ }^{[19,20]} \text {, sudor }{ }^{[21]} \text {, saliva }{ }^{[22]} \text {, metabolitos de drogas en } \\
\text { fluidos fisiológicos }{ }^{[23-25]} \text {, compuestos tóxicos }\end{array}$ \\
\hline Alimentos & $\begin{array}{l}\text { Residuos de pesticidas }{ }^{[27]} \text {, compuestos off-flavor }{ }^{[28-30]} \text { aromatizantes, } \\
\text { saborizantes }^{[31,32]}\end{array}$ \\
\hline $\begin{array}{l}\text { Control de calidad de } \\
\text { productos finales }\end{array}$ & $\begin{array}{l}\text { Polímeros }{ }^{[33,34]} \text {, productos de aseo personal }{ }^{[35]} \text {, } \operatorname{cosméticos}^{[36]} \text {, residuos de } \\
\text { solventes en fármacos }{ }^{[37]} \text {, etc. }\end{array}$ \\
\hline
\end{tabular}


de mil sustancias químicas ${ }^{[40]}$; 2) Los compuestos volátiles (detectables) presentes en mezclas se encuentran en concentraciones muy diferentes; su rango puede ir de $p p t$ hasta $p p m$, o concentraciones más altas ${ }^{[41]}$; 3) La naturaleza química de las sustancias en las mezclas de volátiles es muy diversa, pueden encontrarse simultáneamente tanto moléculas apolares (hidrocarburos), como polares (aldehídos, ácidos, aminas, sulfuros, etc. $)^{[42]}$; se pueden hallar, a menudo, compuestos con heteroátomos, por ejemplo, nitrógeno o azufre $^{[43]}$, cuya detección selectiva demandará el uso de detectores cromatográficos selectivos o específicos.

Los compuestos azufrados, aislados de ajo (bulbos) por la técnica de destilación-extracción simultánea con solvente (SDE) se registran por un sistema de detección con captura de electrones (ECD) selectivamente y con sensibilidad muy alta (Figura 1). Después del consumo de una ensalada con cebolla fresca, en el aliento humano aparecen compuestos azufrados, mayoritariamente sulfuros (mono-, di- y trisulfuros) de alquilos $\left(-\mathrm{C}_{3}\right)$ y alquenilos $\left(=\mathrm{C}_{3}\right)$. En la Figura 2 aparecen perfiles cromatográficos típicos de volátiles presentes en el aliento humano, después del consumo de ensalada, aislados por HS-SPME (PA, $85 \mu \mathrm{m}$ ), y registrados por un detector fotométrico de llama (FPD). Si más bien el FPD no se destaca por la sensibilidad tan alta como la del ECD, su selectividad para detectar compuestos azufrados en una mezcla es excelente.

La diversidad y la cantidad de compuestos volátiles presentes en diferentes matrices es tan alta, que su análisis demanda a veces la combinación de diversas estrategias para su aislamiento (no hay un método "ideal”, único), separación y detección. La base de datos FLAVOR-BASE 2010 (www.Leffingwell.com), por ejemplo, reúne la información sobre la toxicología, estructuras y propiedades físico-químicas de más de 4000 sus-

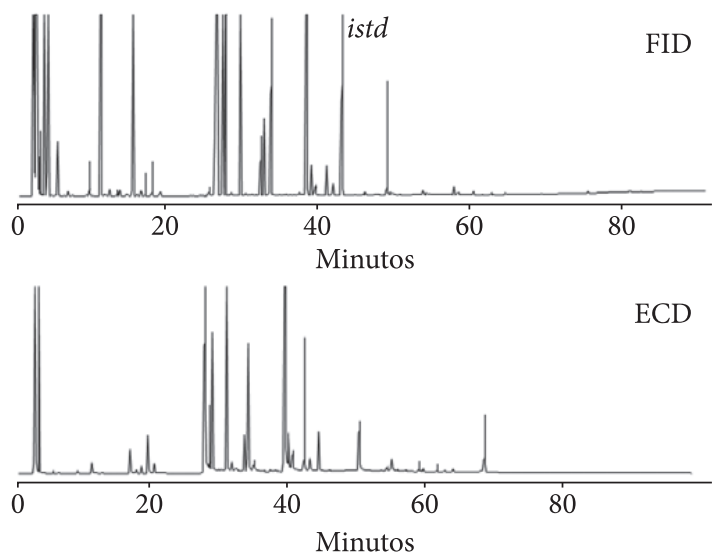

Figura 1 Cromatogramas típicos del extracto de ajo (bulbos), obtenido por destilación-extracción simultánea con solvente (SDE) y analizado en la columna DB-5 (60 m), con diferentes sistemas de detección, a saber: detector de ionización en llama (FID), atenuación de la señal At $=0\left(2^{0}=1\right)$; detector de captura de electrones (ECD), atenuación de la señal At $=5\left(2^{5}=32\right)$. Istd $-n$-tetradecano.

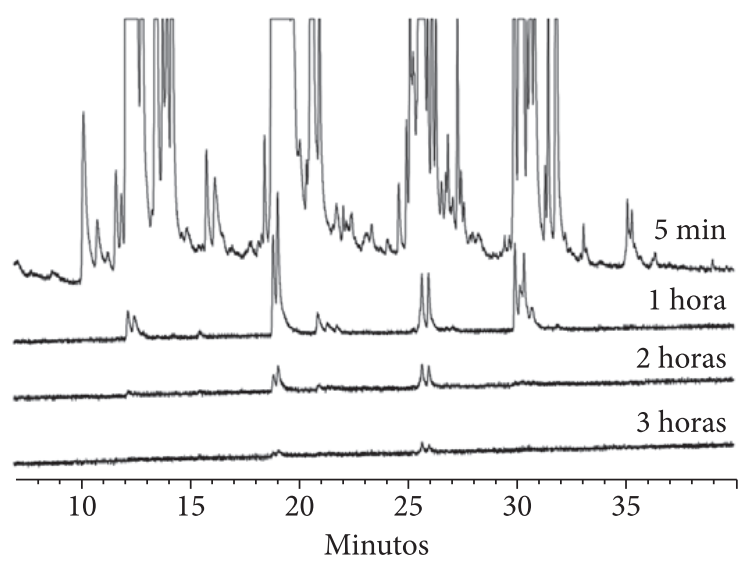

Figura 2 Perfiles cromatográficos típicos de aliento humano, monitoreado por HS-SPME (fibra recubierta con poliacrilato) (PA, $85 \mu \mathrm{m})$; temperatura ambiente, exposición al aliento por 2 minutos), a diferentes intervalos de tiempo después del consumo de ensalada de cebolla. Detector fotométrico de llama (FPD), registro selectivo de compuestos azufrados, mono-, di- y trisulfuros de alquilos $\left(-C_{3}\right)$ y alquenilos $\left(=C_{3}\right)$. Columna HP-5 (60 m).

tancias reconocidas como compuestos-flavor. Los autores, Nijssen y sus colaboradores, reportan en alimentos un número total de compuestos volátiles diferentes mayor de $7000^{[44]}$. 


\section{Aislamiento de compuestos volátiles}

La estrategia para aislar compuestos orgánicos volátiles depende fundamentalmente de: 1) Su naturaleza química; 2) La concentración en la mezcla y sobre todo 3) Del tipo de matriz (estado de agregación, homogeneidad y composición), donde se encuentran. Los experimentos para el monitoreo in vivo de volátiles biogénicos (metabolitos secundarios volátiles de plantas, aliento humano, etc.) conforman un diseño experimental más complejo y esmerado, cuyos aspectos más "frágiles" están relacionados con la cuantificación correcta de los analitos y la reproducibilidad de estas mediciones. Distintos métodos de aislamiento de volátiles pueden producir diferentes composiciones (cuantitativas, pero también cualitativas) de extractos; por ello, a menudo, se usan varias técnicas de extracción para describir lo más completamente posible la composición de la mezcla de volátiles aislada. Es importante tener en cuenta que, para comparar diferentes métodos de extracción incluyendo los aspectos cuantitativos, es necesario usar patrones (estándares) internos, tanto para enriquecer la matriz antes de su extracción, como para agregar a los extractos obtenidos, antes de su inyección al cromatógrafo. Ello dará sentido a la investigación comparativa de diferentes métodos de aislamiento de volátiles.

Entre los métodos más comunes para aislar los compuestos volátiles figuran los de destilación con vapor (SD), la hidrodestilación (HD), la destilación-extracción simultánea con solvente (SDE), la extracción asistida por la radiación de microondas (MWAE), la desorción térmica (TD), el atrapamiento en sorbentes poliméricos y purga y trampa (Purge and Trap, P\&T), la extracción en fase sólida (SPE), la microextracción en fase sólida (SPME), la extracción asistida por membrana, la extracción con fluido supercrítico (SFE), la extracción por sorción en barra de agitación (SBSE) o sobre la cinta recubierta con un sorbente (STE), la microextracción en fase líquida o, simplemente, la transferencia de volátiles directamente al instrumento analítico, e.g., headspace estático, y otros ${ }^{[45,46]}$.

Cuál técnica de extracción se escoge, depende mucho del objetivo del experimento, el tipo de sustancias (target) para extraer, asimismo, de los instrumentos analíticos disponibles y del grado de sensibilidad que se requiere, entre otros aspectos. Por ejemplo, los compuestos carbonílicos volátiles requieren su derivación previa, para ser detectados en la matriz de donde se aíslan, sobre todo, si se encuentran en cantidades a nivel de trazas. El método de extracción no debe generar cambios en el sistema sometido al análisis o monitoreo (e.g., inducir una descomposición térmica o hidrólisis, etc.) y, para la mayoría de aplicaciones, debe ser reproducible, cuantitativo, ojalá evitar el uso de solventes y, que no sea invasivo en sistemas biológicos.

El aislamiento, la detección y la identificación de compuestos carbonílicos volátiles se dificultan debido a las propiedades -muy particulares-, de estas sustancias, a saber: 1) Tienen masas moleculares bajas; 2) Poseen una alta volatilidad; 3) Son parcialmente solubles en agua; 4) Muchos, son reactivos debido a la presencia de grupos aldehído o cetona; 5) Se encuentran a menudo a nivel de trazas y 6) Los detectores convencionales, e.g., TCD, FID o MSD, son poco sensibles para registrarlos de modo reproducible.

Por ejemplo, la detección de acetaldehído, $\mathrm{CH}_{3} \mathrm{CHO}$, en modo directo, por GC-MS, se dificulta adicionalmente por el hecho de que su masa nominal (44 g.mol-1) es igual a la del gas carbónico, $\mathrm{CO}_{2}$, que forma parte del background de muchas matrices, incluyendo el del mismo instrumento de medición, verbigracia, un espec- 
trómetro de masas. Todo ello indica que estas sustancias deben ser derivadas, previa su detección. La derivación de sustancias carbonílicas volátiles permite aumentar su peso molecular, bajar su volatilidad y reactividad y, sobre todo, permite introducir un grupo o varios grupos funcionales, que pueden generan la respuesta más sensible en varios detectores selectivos, e.g., NPD, FPD o ECD (este último, es uno de los más sensibles en cromatografía de gases).

Los productos secundarios finales, aldehídos y cetonas, que se generan durante el proceso de oxidación en lípidos, por ejemplo, en los aceites vegetales, las grasas y las margarinas, pueden ser derivados con pentafluorofenilhidracina (PFPH), formando hidrazonas, cuya detección por ECD, selectiva y sensible (gracias a la presencia de 5 átomos de flúor en la molécula), es además altamente reproducible ${ }^{[47,48]}$. En la Figura 3 aparece un perfil cromatográfico de aldehídos derivados con la PFPH directamente en el aceite de girasol calentado a $220{ }^{\circ} \mathrm{C}$ durante 2 horas, y luego extraídos con solvente.

Uno de los principales productos del metabolismo del alcohol etílico es el acetaldehído, que puede -después del consumo de una bebida alcohólica-, encontrarse tanto en orina y sangre, como en el aliento humano. Su monitoreo por la técnica de HS-SPME en el aliento humano constituye un método sensible, no invasivo y fácil de llevar a cabo. La fibra de SPME (PDMS/DVB, $65 \mu \mathrm{m})$ se satura previamente con el agente derivatizante, i.e., $\mathrm{PFPH}$, y luego, por unos 2 minutos, se expone al aliento humano. La derivación del aldehído acontece in situ, sobre la fibra. Cuando se requiere la cuantificación, se necesita una calibración previa del método. En la Figura 4 aparecen perfiles cromatográficos del acetaldehído (derivado sobre la fibra SPME saturada con $\mathrm{PFPH}$ ), en función del tiempo transcurrido desde la ingestión de una bebida alcohólica (cerveza).

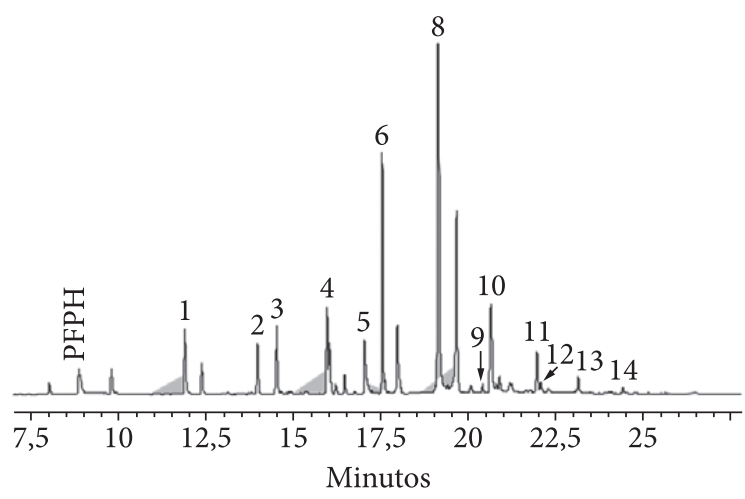

Figura 3 Perfil cromatográfico de aldehídos (derivados con la PFPH) generados en el aceite de girasol (ácido linoleico - 65\%, ácido oleico - 25\%) sometido a calentamiento $\left(22{ }^{\circ} \mathrm{C}\right.$ durante 2 horas). Detector de micro-captura de electrones ( $\mu$-ECD). Columna DB-1 $(60$ m). 1. Acetaldehído; 2. Propanal; 3. Acroleína; 4. Butanal; 5. Crotonaldehído; 6. Pentanal; 7. trans-2-Pentenal; 8. Hexanal; 9. trans-2 Hexenal; 10. Heptanal; 11. trans-2-Heptenal; 12. Octanal; 13. Nonanal; 14. Decanal.

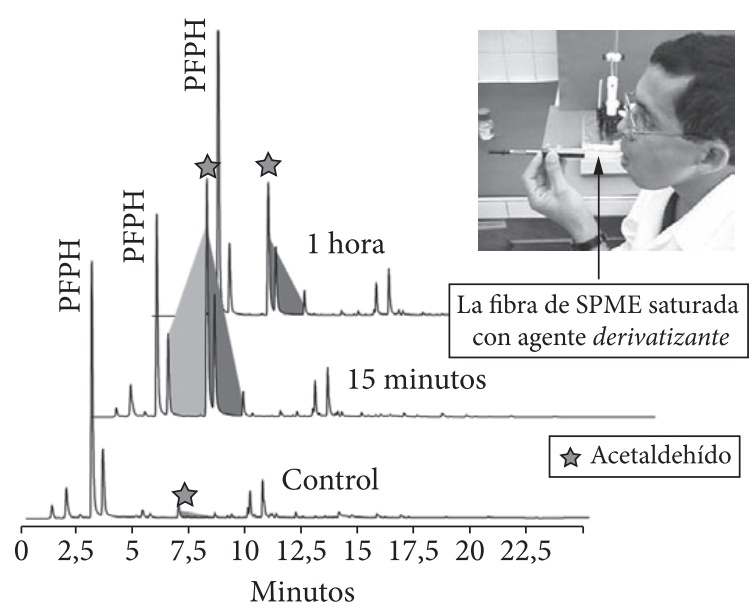

Figura 4 Perfiles cromatográficos típicos del acetaldehído, derivado directamente sobre la fibra de SPME (PDMS/DVB, $65 \mu \mathrm{m}$ ) saturada con PFPH y expuesta al aliento humano (temperatura ambiente, 2 minutos). La detección del acetaldehído se llevó a cabo antes (control) y después (15 minutos, 1 hora) de la ingestión, por un voluntario, de una bebida alcohólica (cerveza). Detector de micro-captura de electrones ( $\mu$-ECD). Columna HP-5 (30 m).

Otro ejemplo es el aislamiento y estudio de volátiles (aroma) de granos de café tostado; para ello, se pueden emplear métodos de headspace dinámico. Por ejemplo, el de purga y trampa 
$(\mathrm{P} \& \mathrm{~T})$, donde un gas $\left(\mathrm{N}_{2}\right)$ barre el espacio encima de los granos de café y arrastra sus compuestos de aroma; éstos se disuelven parcialmente en agua, de la cual se extraen y se concentran en un solvente, e.g., diclorometano, en un arreglo que se asemeja a un extractor líquido-líquido continuo (Figura 5a), según el diseño descrito por Shibamoto y colaboradores ${ }^{[49]}$. El perfil cromatográfico de los volátiles aislados depende tanto de la temperatura de muestreo, como de la duración de la purga (barrido) con el gas inerte; asimismo, del grado de concentración del extracto obtenido y del sistema cromatográfico empleado (modo de inyección, resolución de la columna y sensibilidad del sistema de detección) (Figura 5b).

\section{Aspectos cromatográficos}

\subsection{Inyección de la muestra}

Para el análisis cromatográfico (GC) de una mezcla de sustancias volátiles, virtualmente, se pueden utilizar todos los tipos de inyectores, i.e., el puerto de inyección "clásico" split/splitless, inyectores con temperatura programada (PTV), que, entre otras bondades (e.g., inyección de grandes volúmenes de muestra, LVI), permiten concentrar in situ los analitos en el extracto, evaporando "programadamente" el solvente; los inyectores on-column, que son aptos tanto para sustancias muy volátiles (con un dispositivo de crio-enfocamiento), como para sustancias poco volátiles o inestables térmicamente. El modo de inyección splitless pulsado también puede ser una buena opción para compuestos presentes a nivel de trazas, pero, a la vez, para aquellos que son inestables térmicamente. Cuando se usa el puerto de inyección split/splitless, sobre todo, en el modo split, es importante que la cantidad de analitos que entre a una columna GC sea suficiente y comparable con los niveles mínimos de:
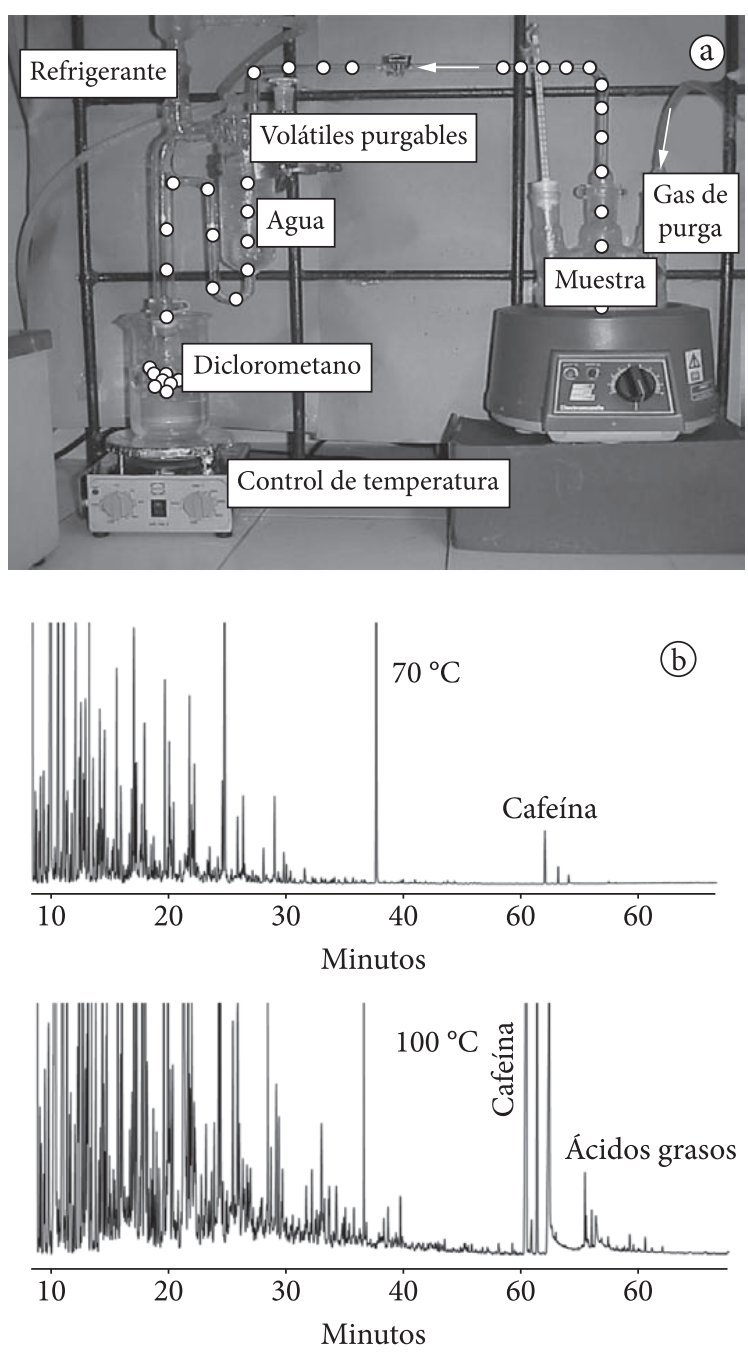

Figura 5 a) Dispositivo de purga con gas $\left(\mathrm{N}_{2}\right)$ y atrapamiento simultáneo en solvente $\left(\mathrm{CH}_{2} \mathrm{Cl}_{2}\right)$ (P\&T) de volátiles de café. b) Perfiles cromatográficos típicos de las fracciones volátiles de los granos de café tostado, obtenidos por el método de P\&T, a diferentes temperaturas $\left(70\right.$ y $\left.100^{\circ} \mathrm{C}\right)$. Duración de purga con $\mathrm{N}_{2}$ - 2 horas. GC-MS. El (70 eV). Columna DB-5MS $(60 \mathrm{~m})$.

1) Detección (si sólo se trata de la necesidad de registrar la presencia de un analito en la muestra); 2) Cuantificación (cuando se requiere la cuantificación exacta de un analito y, para ello, es importante obtener la señal reproducible del detector); 3) Identificación. En este caso, el nivel mínimo de identificación es aquella cantidad mínima del analito que genera una señal espectroscópica, 
verbigracia, un espectro de masas, que cumpla con todos los criterios de calidad; entre ellos, se destacan la presencia de todas las señales isotópicas en el espectro, niveles muy bajos de ruido y de señales intrusas, e.g., de background químico (contaminación, fugas) y la concordancia del espectro obtenido con la estructura química propuesta.

La temperatura del puerto de inyección split/splitless debe ser suficientemente alta para evaporar todos los componentes de la mezcla, pero sin exceder las temperaturas de descomposición. Para el análisis de compuestos volátiles la temperatura del inyector puede estar en el rango de $200-250{ }^{\circ} \mathrm{C}$ (Figura 6a); por supuesto, en las pruebas preliminares hay que encontrar aquella temperatura que conduzca a la mayor sensibilidad (área de la señal cromatográfica). Si el análisis cromatográfico requiere determinaciones cuantitativas y la identificación de analitos se basa en sus índices de retención y espectros de masas (GC-MS), es importante establecer la reproducibilidad de los tiempos de retención y de las áreas cromatográficas. Para esto la misma muestra se inyecta a la columna varias veces (el mismo día y en días diferentes) y se determina la desviación estándar o coeficiente de variación $(\mathrm{CV}, \%)$ de estas mediciones.

\subsection{Columna cromatográfica}

Esta parte del equipo es fundamental para el análisis de mezclas multicomponente, ya que permite vislumbrar el número mínimo de componentes presentes, puesto que algunos pueden presentar co-eluciones. Cuántos analitos, en realidad se encuentran en una mezcla (un extracto) depende de varios parámetros, a saber: 1) La preparación del extracto (grado de su concentración); 2) El modo de inyección (split, splitless, on-column, PTV); 3) La capacidad que tenga la
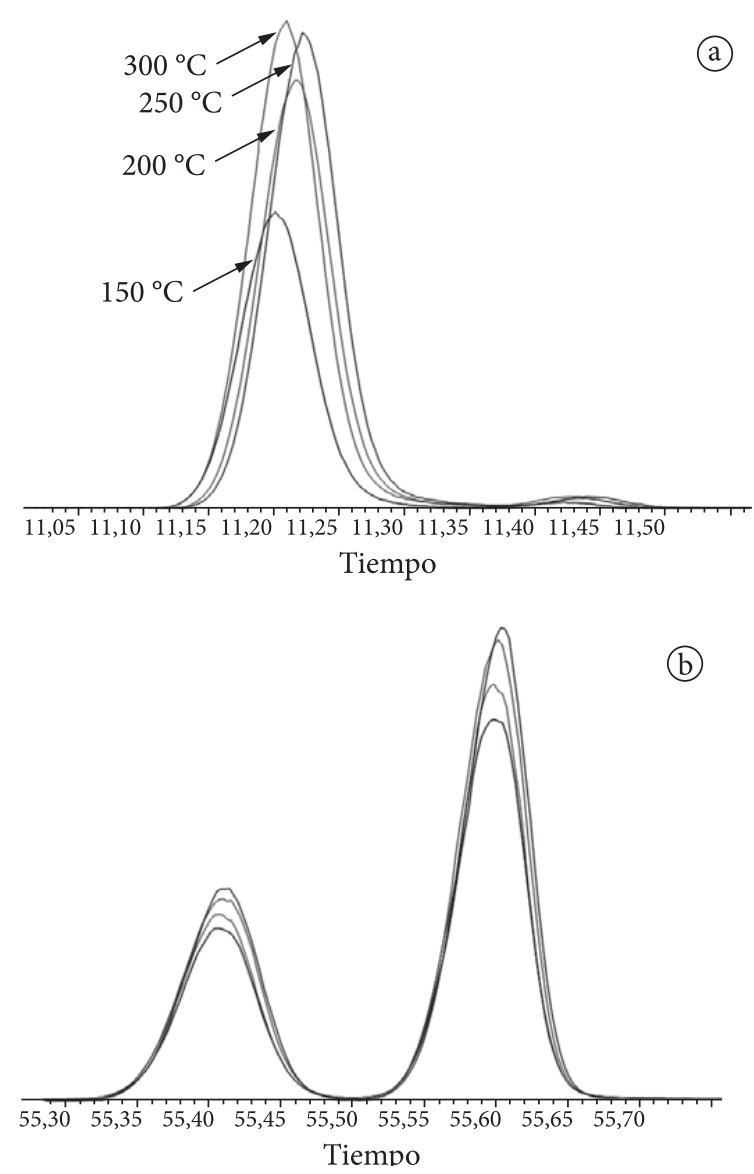

Figura 6 a) Las áreas cromatográficas del $\alpha$-pineno (monoterpeno con temperatura de ebullición de $155{ }^{\circ} \mathrm{C}$ ) inyectado a la columna cromatográfica en el modo split (1:30), a diferentes temperaturas del puerto de inyección (150, 200, 250 y $300{ }^{\circ} \mathrm{C}$ ); b) Reproducibilidad de tiempos de retención y áreas cromatográficas de óxido de cariofileno ( $t_{R}=55.4$ minutos) y (Z)-nerolidol ( $t_{R}=55.6$ minutos), analizados por GC-MS, en diferentes días de la semana, en la columna DB-5MS (60 m). El (70 eV).

columna cromatográfica de separar completamente los componentes de la mezcla y 4) La sensibilidad del sistema de detección empleado.

Ya que las mezclas o extractos de compuestos orgánicos volátiles pueden estar conformadas por una cantidad grande de componentes (decenas y hasta centenares) y, además, muchos de ellos pueden ser isómeros (de posición, geométricos, estereoisómeros), se emplean para su análisis columnas cromatográficas largas de 
50 ó $60 \mathrm{~m}$; para análisis de gasolinas, de $100 \mathrm{~m}$ de longitud. El diámetro interno (D.I.) de la columna se escoge de acuerdo con la cantidad de la muestra a inyectar (a menudo, se requiere incrementar la cantidad de analitos introducidos a la columna), pero típicamente se usan columnas de 0.25 ó $0.32 \mathrm{~mm}$ de D.I. (drogas, pesticidas, residuos de explosivos), aunque las columnas de menor diámetro $(0.18,0.20$ ó $0.22 \mathrm{~mm})$ permiten lograr una eficiencia de separación mucho más alta, pero comprometiendo, en algunos casos, la sensibilidad (capacidad de carga de la columna). Un parámetro importante es el grosor de la fase estacionaria $\left(\mathrm{d}_{\mathrm{f}}\right)$, que, para el análisis cromatográfico de compuestos volátiles, debe ser mayor (comúnmente, se usan columnas de $0.25 \mu \mathrm{m}, \mathrm{d}_{\mathrm{f}}$ ) y, para analitos muy volátiles, puede llegar hasta $5 \mu \mathrm{m}$. La polaridad de la fase estacionaria se escoge de acuerdo con la polaridad de los analitos, pero, en el caso de mezclas "heterogéneas", por ejemplo, aceites esenciales, que contienen analitos tanto polares como apolares, es necesario (obligatorio!) usar ambas columnas, i.e., polar (f.e. de polietilenglicol) y apolar (100\%-polidimetilsiloxano ó 5-95\%-fenil polimetilsiloxano).

La identificación (presuntiva) de los analitos se basa en los índices de retención lineales medidos en dos columnas "ortogonales" ${ }^{[50]}$, y en los espectros de masas (EI, $70 \mathrm{eV}$, patrones de fragmentación) ${ }^{[51]}$. La confirmación de la identidad química de una sustancia se hace usando compuestos-patrón, que son imprescindibles también para la cuantificación de analitos por medio de curvas de calibración. Para la cuantificación, se pueden usar métodos de estandarización externa, interna o de adición de compuesto estándar. Frecuentemente, para el análisis de aceites esenciales se reportan cantidades relativas (\%) de los componentes presentes en el aceite. En la Figura 7 aparecen dos perfiles cro-

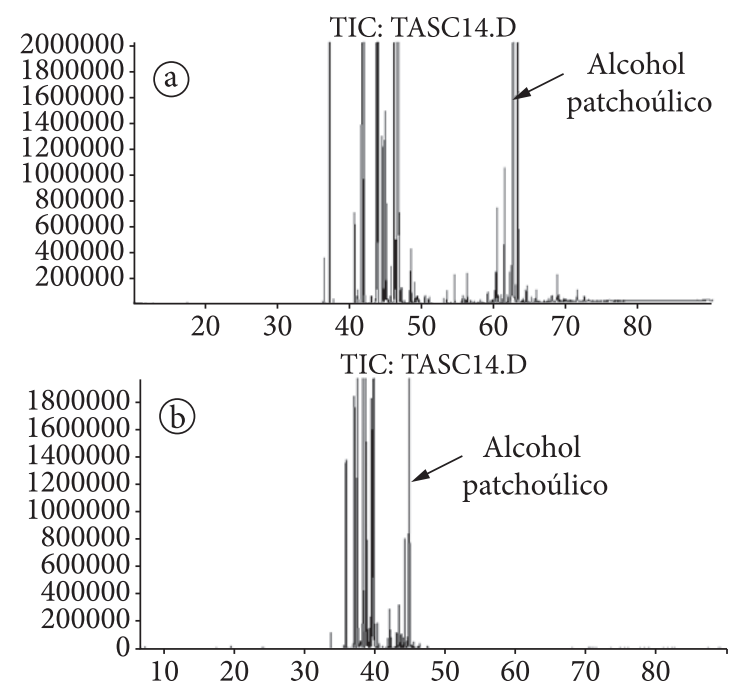

Figura 7 Perfiles cromatográficos típicos obtenidos por GC-MS (El, 70 eV) del aceite esencial de patchoulí (Pogostemon cablin, familia Labiatae) en: a) Columna con la fase estacionaria polar (DB-WAX, 60 m, polietilenglicol) y b) Columna apolar (DB-5, 60 m, 5\%-fenil-polimetilsiloxano).

matográficos del aceite esencial, hidrodestilado de las hojas de patchoulí (Pogostemon cablin, familia Labiatae), y analizado en columnas polar y apolar. La diferencia de los dos perfiles es muy notoria, pero en ninguna de las dos columnas se logran separar completamente todos los componentes de esta compleja mezcla. La identificación completa de algunos sesquiterpenoides presentes en el aceite de patchoulí se dificulta porque las respectivas sustancias-patrón no están disponibles y, además, en varios casos, se presenta la co-elución de sustancias, unas en la columna polar, otras, en la apolar. La cromatografía total o exhaustiva (Comprehensive GCxGC) sería una solución para este último inconveniente: esta técnica comprende la unión de una columna analítica estándar (25-30 m), a través de un modulador, con una columna de cromatografía rápida (muy corta, ultra-delgada) con la fase estacionaria "ortogonal" (polar-apolar o apolar-polar). 


\subsection{Sistemas de detección}

En muchos extractos de compuestos orgánicos volátiles se pueden encontrar diferentes familias de sustancias, entre ellas, las que tienen heteroátomo(s). El ejemplo típico es el aroma de café, que, junto con muchos otros, contiene compuestos nitrogenados (derivados de piridina, piperazinas, oxazoles, pirroles, etc.) y azufrados (derivados de tiofeno y tetrahidrotiofeno), productos de la reacción de Maillard. La combinación de sistemas de detección selectivos (e.g., NPD y FPD) con el detector de masas (MSD), ayuda mucho a la identificación química de componentes de la mezcla compleja (Figura 8). Algunas sustancias nitrogenadas o azufradas pueden co-eluir en la columna cromatográfica con aquellos compuestos que no generan respuesta en detectores selectivos. Sus espectros de masas no son "homogéneos", ya que se vislumbra en ellos la superposición de señales de distintas moléculas. Aunque se pueden emplear los programas computaciones especiales para revisar la "pureza" del pico cromatográfico (peak purity) o para realizar la deconvolución de espectros, la presencia de nitrógeno o azufre en una molécula se confirmará inequívocamente sólo usando los respectivos detectores selectivos. La solución "total" para separar la mezcla compleja de volátiles -con un número muy alto de componentes, presencia de estructuras isoméricas, con heteroátomos y compuestos a nivel de trazas, etc.-, sería el uso de la cromatografía exhaustiva (Comprehensive GCxGC), en combinación con un analizador másico de barrido (scan) muy rápido y de alta resolución, para determinar la composición elemental de los iones (e.g., analizador de tiempo de vuelo, TOF, con reflectrón, o espectrometría de masas de ion-ciclotrón con transformada de Fourier, FT-ICR-MS), pero... ¡vaya la accesibilidad y costo de estos instrumentos!

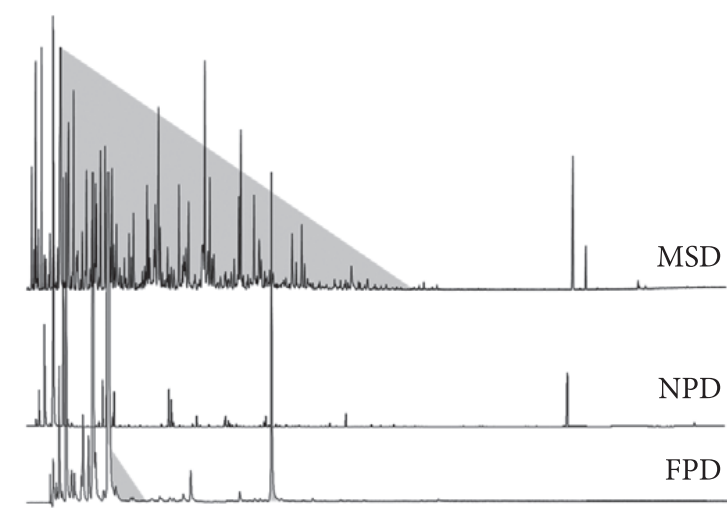

Figura 8 Perfiles cromatográficos típicos de la fracción volátil (aislada por la técnica P\&T de granos de café tostado), obtenidos en la columna HP-5 (50 $\mathrm{m} \times 0.25 \mathrm{~mm} \times 0.33 \mu \mathrm{m})$ con diferentes sistemas de detección, MSD (El, 70 eV), NPD y FPD.
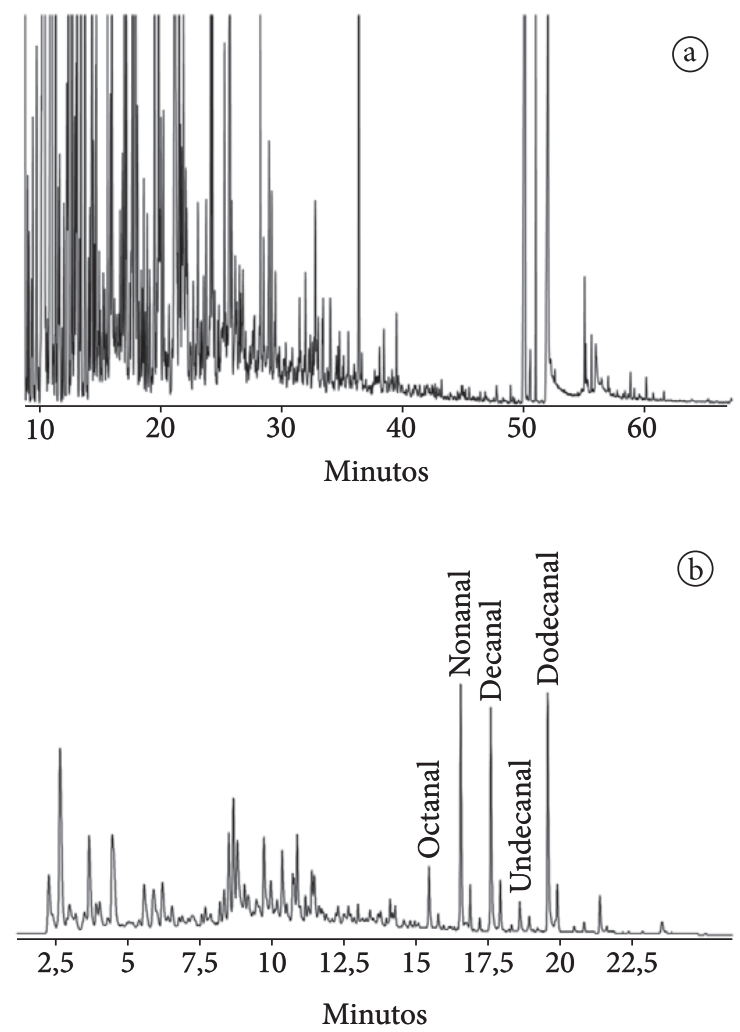

Figura 9 a) Corriente iónica total obtenida por GC-MS (El, 70 eV) de la fracción volátil de granos de café tostado (aislada por el método P\&T). b) Perfil cromatográfico de aldehídos derivados con PFPH in situ sobre la fibra de SPME expuesta al espacio de cabeza (headspace) de granos de café tostado. Detector de captura de electrones (ECD). Columna DB-5 (60 m). 
Sería un trabajo verdaderamente titánico descubrir la presencia de compuestos carbonílicos, de peso molecular bajo y mediano, en una corriente iónica total (=cromatograma), obtenida por GC-MS (EI, $70 \mathrm{eV}$ ) de la fracción volátil -aislada por P\&T- de granos de café tostado (Figura 9a). La dificultad consiste en que muchos de estos compuestos se encuentran a nivel de trazas, algunos, co-eluyen con otras sustancias, mientras que en los espectros de masas de varios aldehídos alifáticos y cetonas frecuentemente no se observan señales de iones moleculares. Sobre una fibra de SPME, saturada previamente con el agente derivatizante (i.e., $\mathrm{PFPH}$ ) y expuesta a la fase vapor de café (granos tos- tados), los aldehídos y cetonas, presentes en el aroma de café, reaccionarán in situ formando las respectivas hidrazonas, que luego se desorben en el puerto de inyección de GC y se registran por el sistema de detección altamente sensible, ECD (Figura 9b). La identificación de compuestos carbonílicos se basa en la comparación de sus tiempos de retención y espectros de masas con los de las sustancias-patrón (hidrazonas sintetizadas).

Para el desarrollo del método GC-MS para análisis de compuestos orgánicos volátiles, es importante tener en cuenta varios aspectos $y$ "normalizar" diferentes parámetros, algunos de los cuales aparecen descritos en la Figura 10.
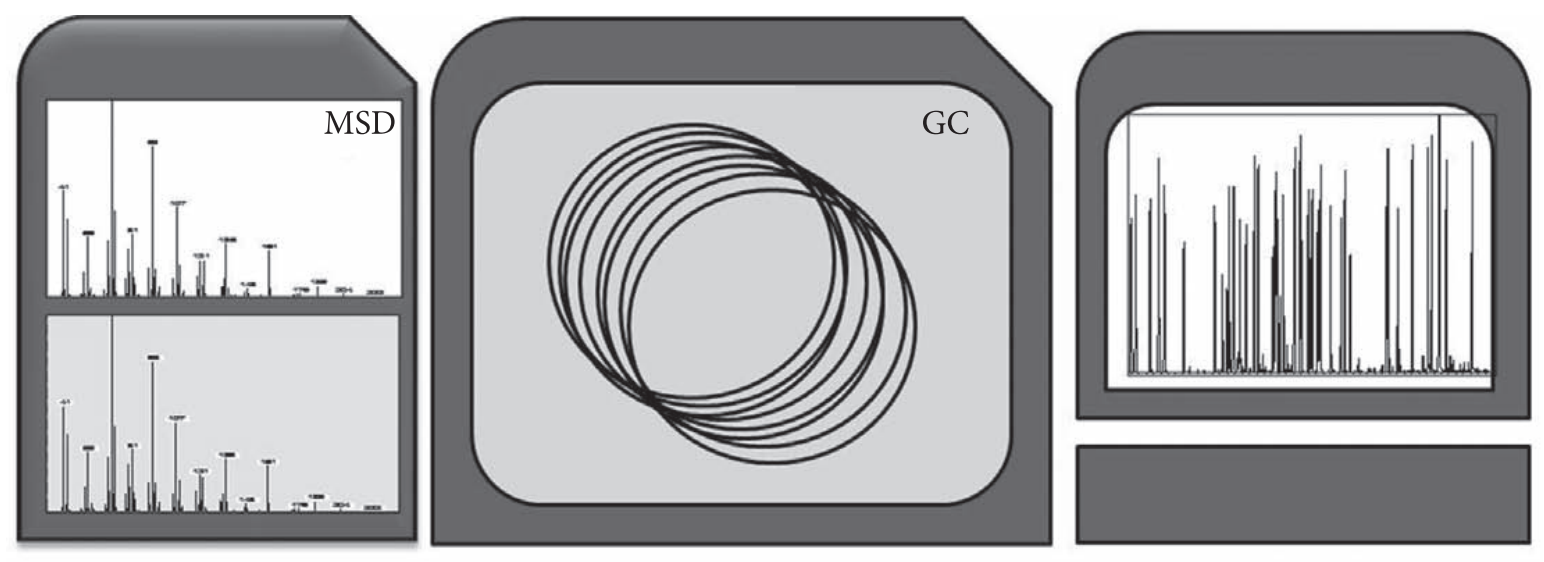

\begin{tabular}{|c|c|c|}
\hline Detector de masas & Cromatógrafo & Sistema de datos \\
\hline Modo de ionización & Modo de inyección & Software \\
\hline Analizador $(\mathrm{es})(\mathrm{MS} / \mathrm{MS})$ & Selección de la columna & $\begin{array}{c}\text { Velocidad de aquisición, } \\
\text { deconvolución }\end{array}$ \\
\hline $\begin{array}{c}\text { Modo de aquisición } \\
(\text { SIM, scan, MS/MS) }\end{array}$ & $\begin{array}{c}\text { L } \times \text { D.I. } \times \mathrm{d}_{\mathrm{p}} \text { polaridad de } \\
\text { la fase estacionaria }\end{array}$ & $\begin{array}{c}\text { Bases de datos de espectros } \\
\text { Índices de retención }\end{array}$ \\
\hline Especificidad y resolución & Programación de temperatura & Reporte \\
\hline
\end{tabular}

Mantenimiento preventivo y correctivo

Atualización

Figura 10 Esquema general del sistema GC-MS y varios aspectos y parámetros -a tener en cuenta-, para su desempeño eficiente. 

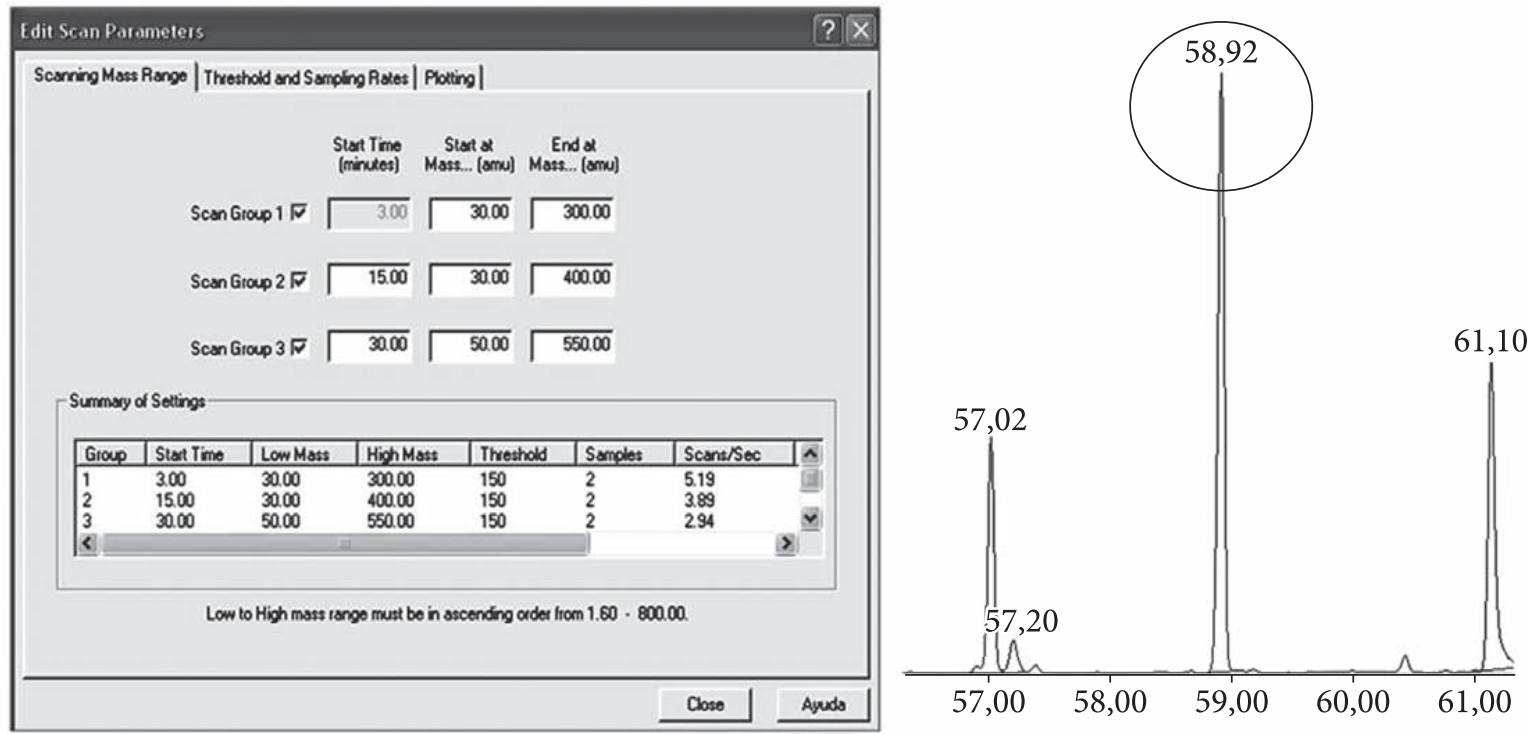

\begin{tabular}{|c|c|c|}
\hline Masa baja & Masa alta & Scan/segundos \\
\hline 30 & 300 & 5,19 \\
\hline 30 & 400 & 3,89 \\
\hline 50 & 550 & 2,94 \\
\hline
\end{tabular}

\begin{tabular}{|c|c|}
\hline$t_{\mathrm{R}}$ & 58,92 minutos \\
\hline Compuesto & Guaiol (P.M. 222) \\
\hline Rango de masas & $\begin{array}{c}\text { Número de barridos } \\
\text { registrados en el ápice }\end{array}$ \\
\hline$m / z$ 40-280 & 8549 \\
\hline$m / z$ 40-550 & 16895 \\
\hline
\end{tabular}

Figura 11 Relación entre el rango de masas (masas mínima y máxima) y el número de barridos (scans = espectros de masas) registrados por unidad de tiempo. El número de scans adquiridos en el ápice del pico cromatográfico correspondiente a sesquiterpenol, guaiol $\left(\mathrm{C}_{15} \mathrm{H}_{26} \mathrm{O}, \mathrm{t}_{R}=58.92\right.$ minutos) es casi dos veces mayor para el intervalo de masas m/z 40-280, que para el de m/z 40-550. GC-MS. Detector selectivo de masas Agilent Technologies MSD 5975, El, $70 \mathrm{eV}$.

La parte cromatográfica de GC-MS es prácticamente la misma que se usa para el sistema GC convencional; se necesita considerar los siguientes aspectos, a saber: 1) la selección correcta de la columna; 2) sus dimensiones ( $\mathrm{x}$ x D.I. $\mathrm{x} \mathrm{d}_{\mathrm{f}}$ ); 3) la polaridad de la fase estacionaria; 4) el modo de inyección y 5) la programación de temperatura de horno. Las restricciones para el método GC-MS son el diámetro interno de la columna (generalmente, igual o menor de $0.25 \mathrm{~mm}$ ) y la naturaleza de la fase estacionaria, que debe ser "fortalecida", es decir, más resistente a las temperaturas altas y con un menor nivel de sangrado (bleeding); este último puede afectar drástica- mente el desempeño del espectrómetro de masas, produciendo su contaminación, conducente a la pérdida de sensibilidad, y el deterioro de la calidad de los espectros de masas, por la aparición en ellos de señales intrusas (background).

Cuando se desarrolla un método cuantitativo o se comparan las corrientes iónicas totales $(\mathrm{TIC}=$ cromatograma) de una serie de muestras o se construye una curva de calibración, hay que considerar varios aspectos para la operación correcta del detector selectivo de masas (MSD). Para alcanzar la reproducibilidad de mediciones más alta, es importante mantener la temperatura constante de la 

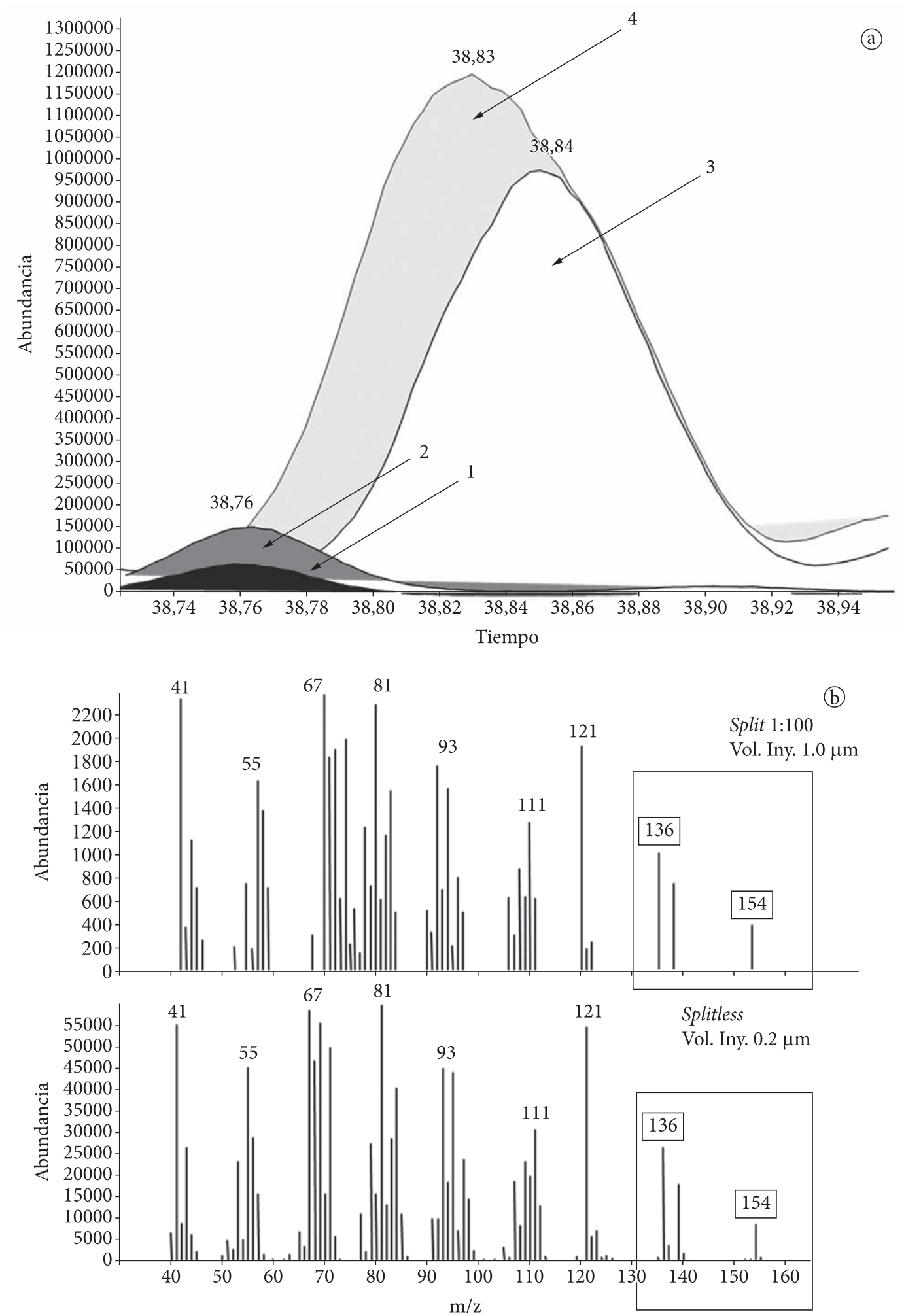

Figura 12 a) Picos cromatográficos (GC-MS, El, $70 \mathrm{eV}$ ) del isopulegol presente en un aceite esencial inyectado en modos: 1) split 1:100;2) split 1:30;3) splitless; 4) pulsed splitless. b) Espectros de masas del isopulegol obtenidos de la corriente iónica total de un aceite esencial inyectado en modos split (1:100) y splitless. Solo el segundo espectro (modo de inyección splitless) cumple con criterios de calidad del espectro de masas y descubre la presencia de todas las señales isotópicas (véanse señales en $\mathrm{m} / \mathrm{z} 136,139$ y 154). 
Tabla 2 Descripción de las once muestras de Pisco peruano de diferencies localidades, cuyas fracciones volátiles fueron aislados por HS-SPME y analizadas por GC-MS.

\begin{tabular}{cccccc}
\hline$N^{\circ}$ & Pisco & Tipo & Valle & Marca & Descripción suministrada \\
\hline 1 & Albilla & Puro & Chincha & Viña Oro & - \\
2 & Italia & Mosto verde & Chincha & Viña Oro & Fermentación interrumpida* \\
3 & Italia & Puro & Chincha & Viña Oro & - \\
4 & Mollar & Puro & lca & Viejo Tonel & No aromático \\
5 & Moscatel & Puro & Chincha & Viñas Oro & - \\
6 & Negra Criolla & Puro & Chincha & Viñas Oro & No aromático \\
7 & Quebranta & Mosto verde & Chincha & Viñas Oro & No aromático \\
8 & Quebranta & Puro & Chincha & Viñas Oro & No aromático \\
9 & Torontel & Mosto verde & Chincha & Viñas Oro & Fermentación interrumpida* \\
10 & Torontel & Puro & Chincha & Viñas Oro & Fermentación interrumpida* \\
11 & Uvina & Puro & Mala & Gran Cruz & No aromático \\
\hline
\end{tabular}

*Se destila con un poco de azúcar remanente.

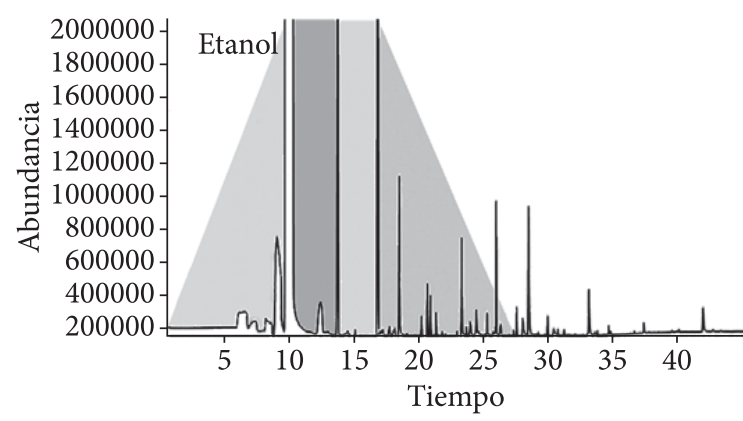

Figura 13 Perfil cromatográfico típico (TIC, El, 70 eV) de la fracción volátil de Pisco, aislada por la técnica de HS-SPME/GC-MS. Columna DB-WAX (60 m).

cámara de ionización $\left(200-300{ }^{\circ} \mathrm{C}\right)$, de la línea de transferencia (Columna $\rightarrow$ Cámara de ionización, $280-310^{\circ} \mathrm{C}$ ) y del analizador de masas (150-200 ${ }^{\circ} \mathrm{C}$ ), durante el análisis de todas las muestras a comparar o de patrones de calibración. Es importante evitar fugas en el sistema, hay que supervisar la presión residual $\left(10^{-5}-10^{-6}\right.$ Torr $)$ en el espectrómetro de masas y calibrarlo (tune) frecuentemente, para poder registrar oportunamente los cambios en su desempeño (e.g., aumento brusco del voltaje de electromultiplicador, etc.) o una posible contaminación.
Para el diseño del método de adquisición, por GC-MS, hay que tener en cuenta lo siguiente: 1) El modo de ionización: para la técnica de GC-MS es más frecuente el uso de impacto de electrones (EI, $70 \mathrm{eV}$ ), aunque también se emplea -para algunas muestras-, la ionización química (CI) de iones positivos (PI) o de iones negativos (NI); por ejemplo, para determinar la masa de una molécula cuyo ion molecular, debido a su labilidad o inestabilidad, no se registra en el espectro obtenido por EI, pero sí aparecerá en los espectros obtenidos por métodos "blandos" de ionización (PI CI o NI CI, según la naturaleza de la molécula); 2) $E l$ modo de adquisición, barrido completo (full scan) o monitoreo de ion(es) seleccionado(s) (SIM); si se tiene el sistema tándem (MS/MS), se pueden emplear diferentes modos de monitoreo a) de un ion precursor, b) de fragmentos filiales, c) iones con la pérdida de un fragmento neutro común o d) monitoreo de una reacción específica, verbigracia, el proceso de transición entre los dos iones, $\mathrm{A}^{+} \rightarrow \mathrm{B}^{+}$, cuyo registro puede elevar sobremanera la especificidad de la detección de un analito target en una mezcla muy compleja, con bastantes interferencias químicas ${ }^{[52]}$. 


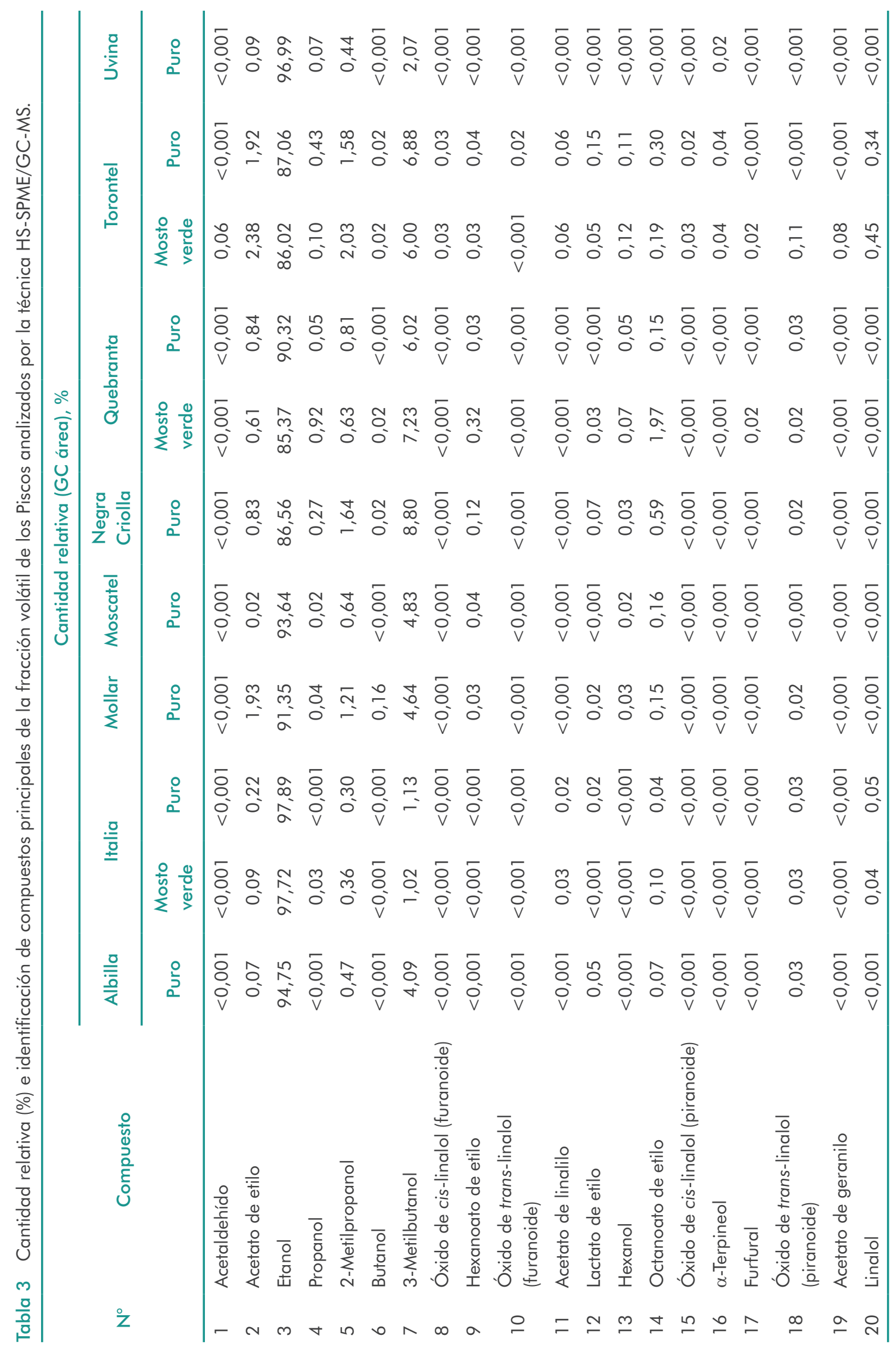




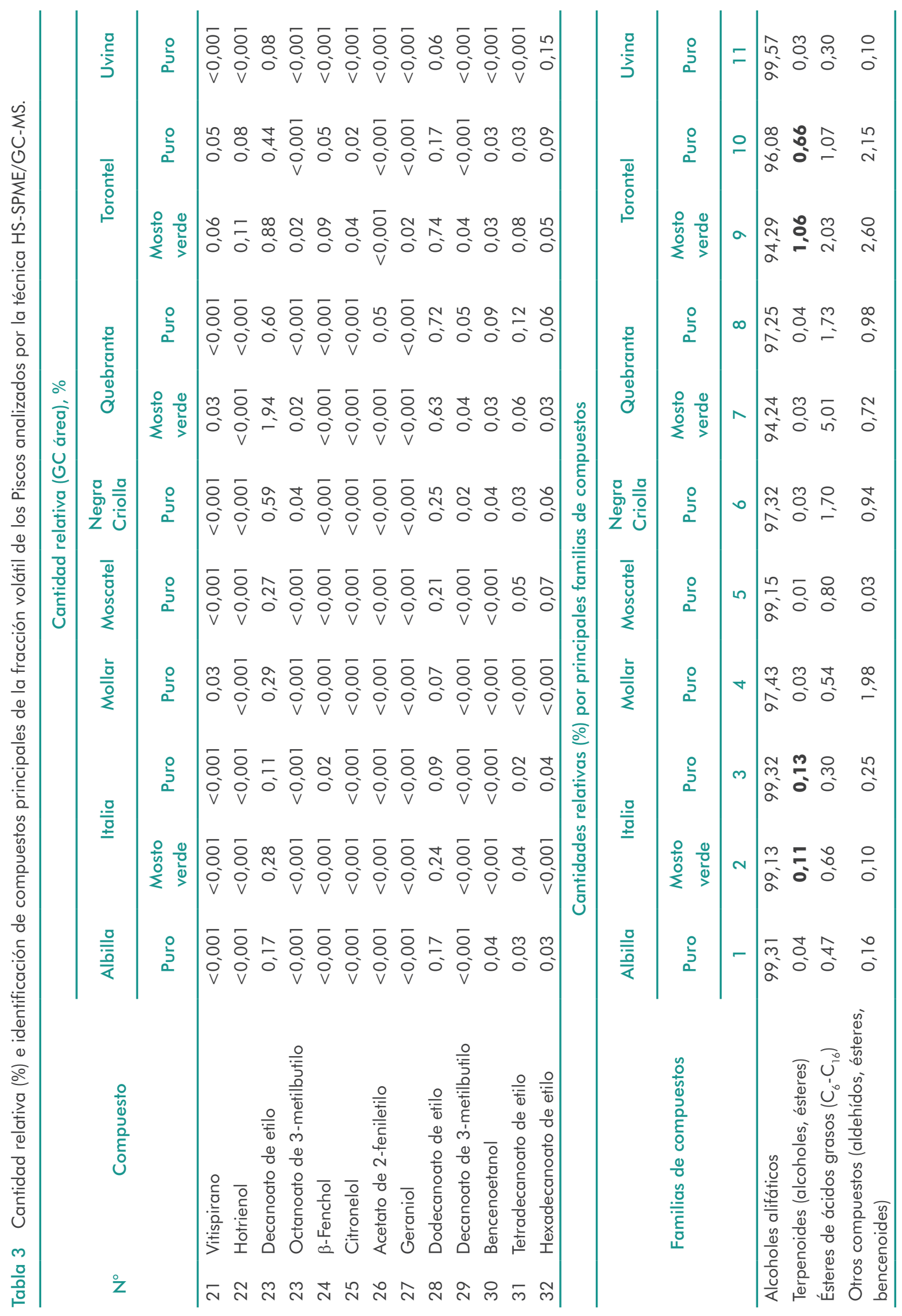


Para la adquisición de la corriente iónica, en función del tiempo, es importante establecer un rango (intervalo) de masas -mínima y máxima-, ya que de esto dependerá el número de espectros obtenidos por unidad de tiempo. En la Figura 11 aparece la relación entre el rango de masas y el número de barridos (scans) obtenidos. A mayor rango de masas, el numero de espectros de masas (scans) obtenidos por unidad de tiempo será menor; el número de barridos es muy importante para la "reconstrucción" de un pico cromatográfico con base en la corriente iónica obtenida y reconstruida.

En numerosas publicaciones científicas, se puede encontrar a menudo, que se usa un rango de masas $(\Delta \mathrm{m})$ de $m / z$ 30-550 para análisis de sustancias volátiles, por GC-MS y cuyos pesos moleculares no superan 250-280 Da (por ejemplo, aceites esenciales). La masa máxima del intervalo $\Delta \mathrm{m}$ podría ser el peso molecular del compuesto "más pesado" presente en una mezcla (extracto) mas unas 40-50 unidades; mientras que la masa mínima depende del tipo de sustancias, porque, por ejemplo, los alcoholes y aminas, en sus espectros de masas, registran fragmentos, respectivamente, en $\mathrm{m} / z 31 \mathrm{y}$ 30, que son estructuralmente relevantes para su identificación. Para compuestos aromáticos, la masa mínima en $\mathrm{m} / \mathrm{z} 50$ puede ser adecuada para empezar los barridos (scans). En la región de masas livianas, se pueden encontrar las señales de gases $\left(\mathrm{N}_{2}, \mathrm{O}_{2}, \mathrm{CO}, \mathrm{Ar}, \mathrm{CO}_{2}\right)$ y de solventes remanentes presentes en la cámara de ionización, mientras que en la región de masas más pesadas prevalecen señales intrusas (exógenas, no propias de una molécula de analito) debido al sangrado de la fase estacionaria de la columna o iones provenientes de la contaminación de diferentes partes del equipo (inyector, columna, cámara de ionización).
Otro aspecto importante está relacionado con la calidad del espectro de masas obtenido, que es importante para identificar el compuesto con base en su patrón de fragmentación o por comparación con el espectro de masas de la base de datos o con el espectro de la respectiva sustancia-patrón. En la Figura 12a aparece el pico cromatográfico de isopulegol registrado, presente en una concentración relativa menor del $0.005 \%$; en los cromatogramas (aparece sólo el pico del isopulegol) presente en un aceite esencial, inyectado en modo split (relación 1:100 y 1:30), splitless y pulsed splitless. La cantidad máxima del isopulegol ingresa a la columna cuando la mezcla se inyecta en modo pulsed splitless. Los espectros de masas del isopulegol obtenidos cuando la muestra se inyecta en modos splitless o pulsed splitless cumplen con los criterios de calidad del espectro de masas, mientras que los obtenidos de corrientes iónicas totales de muestras inyectadas en modo split, carecen de varias señales (por ejemplo, picos isotópicos de los iones en $m / z 136,139$ y 154) (Figura 12b).

\section{Algunos ejemplos}

\subsection{Análisis de fracciones volátiles de Piscos}

Para dar valor agregado y científico a muchos productos agrícolas, evaluar su calidad y aumentar su competitividad en los mercados, es necesario hacer su caracterización completa, establecer propiedades físico-químicas, composiciones químicas y, en el caso de algunos productos, tales como vinos $\mathrm{u}$ otras bebidas alcohólicas, se requiere a menudo certificar su denominación de origen. El estudio cromatográfico de aromas de vinos y otros spirits se ha empleado tradicionalmente como herramienta para establecer su origen, calidad y autenticad, entre otros aspectos. 
Tabla 4 Descripción de las notas odoríferas de algunos componentes presentes en las fracciones volátiles (aroma) de Piscos analizados.

\begin{tabular}{|c|c|}
\hline Sustancia & Descripción organoléptica* \\
\hline (R)-(-)-Linalol & Floral, woody lavender note. \\
\hline$(S)-(+)$-Linalol & Sweet, floral; odor reminiscent of petit-grain and lavender. \\
\hline$(3 R)-(+)-C i t r o n e l l o l$ & Citronella, oil-like, slightly oily, light rosy-leafy, petal-like. \\
\hline (3S)-(-)-Citronellol & $\begin{array}{l}\text { Floral, rose-like odor, reminiscent of geranium oil, } \\
\text { citronellal-like odor with woody aspect. }\end{array}$ \\
\hline$(3 S)-(+)$-Hotrienol & Green fresh flower aroma, graceful and sweet. \\
\hline (3R)-(-)-Hotrienol & Citrus fruit-juice aroma with a pure sweet flower aroma. \\
\hline (3R,6R)- Óxido de cis-linalol (Pyranoide) & Earthy. \\
\hline (3S,6S)- Óxido de cis-linalol (Pyranoide) & Sweet, floral, creamy. \\
\hline (3R,6S)- Óxido de trans-linalol (Pyranoide) & Sweet, floral, creamy. \\
\hline (3S,6R)- Óxido de trans-linalol (Pyranoide) & Earthy. \\
\hline (2R,5R)- Óxido de trans-linalol (Furanoide) & Leafy, earthy. \\
\hline (2S,5S)- Óxido de trans-linalol (Furanoide) & Sweet, floral, creamy. \\
\hline (2S,5R)- Óxido de cis-linalol (Furanoide) & Sweet, floral, creamy. \\
\hline (2R,5S)- Óxido de cis-linalol (Furanoide) & Leafy, earthy. \\
\hline (S)-(-)- $\alpha$-Terpineol & Coniferous odor character, tarry. \\
\hline$(R)-(+)$ - $\alpha$-Terpineol & Heavy floral, typically lilac odor. \\
\hline Hexanoato (Caproato) de etilo & Banana, pineapple. \\
\hline Octanoato (Caprilato) de etilo & Floral, fruity, sweet, vinous, waxy. \\
\hline Dodecanoato (Laureato) de etilo & Fruity, floral, soapy, sweet, waxy. \\
\hline Tetradecanoato (Miristato) de etilo & Waxy, soapy. \\
\hline Hexadecanoato (Palmitato) de etilo & Waxy, fruity, creamy \\
\hline
\end{tabular}

*Nota: Para una mayor precisión, se conservó el nombre en inglés de las notas aromáticas, tal como lo registran las bases de datos consultadas: http://www.leffingwell.com/chirality/, http://www.thegoodscentscompany.com/data/

La combinación de la técnica de extracción de SPME en el modo headspace y el análisis por GC-MS de fracciones volátiles fue aplicada para distinguir los Piscos provenientes de diferentes localidades de Perú (Tabla 2).

El estudio de la composición química de las fracciones volátiles de los once Piscos, aisladas por HS-SPME [fibra de poli(acrilato), PA, de $65 \mu \mathrm{m}$ de grosor, $40^{\circ} \mathrm{C}, 10$ minutos)] y analizadas por GC-MS, reveló la ocurrencia de un total de 32 sustancias (en cantidades relativas $>0.001 \%$ ). Entre éstas, predominaron alcoholes alifáticos (básicamente, etanol), ésteres de ácidos grasos,
$\mathrm{C}_{6}-\mathrm{C}_{16}$, y terpenoides. El perfil cromatográfico típico de la fracción volátil de Pisco aparece en la Figura 13, mientras que en la Tabla 3 se registra la información sobre composición química de las fracciones volátiles de las bebidas analizadas y la cantidad relativa (\%) tanto de las sustancias individuales, como de las principales familias de compuestos presentes. Estos resultados revelan que las fracciones volátiles de las bebidas descritas como "no aromáticas" (Véase la Tabla 2) poseen una cantidad relativa (\%) de terpenoides más baja que e.g., los Piscos identificados con los números 2, 3, 9 y 10. 
En la Tabla 4 se resume la información sobre las notas odoríferas de algunos compuestos presentes en las fracciones volátiles de los Piscos analizados. Es importante anotar, que el impacto odorífero (nota organoléptica, sensorial, y el umbral de su percepción) es función de la configuración enantiomérica de la sustancia (isomería óptica), y de su concentración en la mezcla. Por ello, para el estudio mucho más completo de aromas, se requiere no solamente hallar cantidades relativas (\%), sino también cantidades exactas de componentes presentes, junto con el establecimiento de su configuración óptica, ya que de estos parámetros finalmente dependerá la descripción completa del bouquet aromático de una bebida fermentada. Aún así, la información composicional obtenida permitió establecer diferencias y similitudes de fracciones volátiles de los Piscos analizados, ya que conforma una base objetiva para diferenciarlos. Los datos se sometieron al análisis de componentes principales (PCA), cuyo resultado aparece en la Figura 14 y permite vislumbrar las semejanzas composicionales de las fracciones volátiles (aromas) y clasificar las once muestras de Pisco analizadas, por grupos (clusters), que descubren la mayor similitud de sus fracciones volátiles. Las muestras $\mathrm{N}^{\circ} 9$ y 10 (Pisco Torontel) presentaron las mayores diferencias composicionales con respecto a otros Piscos analizados.

\subsection{Análisis de drogas de síntesis}

Indudablemente, la técnica de GC-MS tiene una gran importancia para el análisis de drogas de síntesis o de diseño (street drugs), ya que sus extractos frecuentemente son mezclas multicomponente, a menudo de composición desconocida y compleja. Precisamente, es cuando el poder de la espectrometría de masas -como método para elucidar estructuras químicas con base en los patrones de fragmentación o por comparación de sus

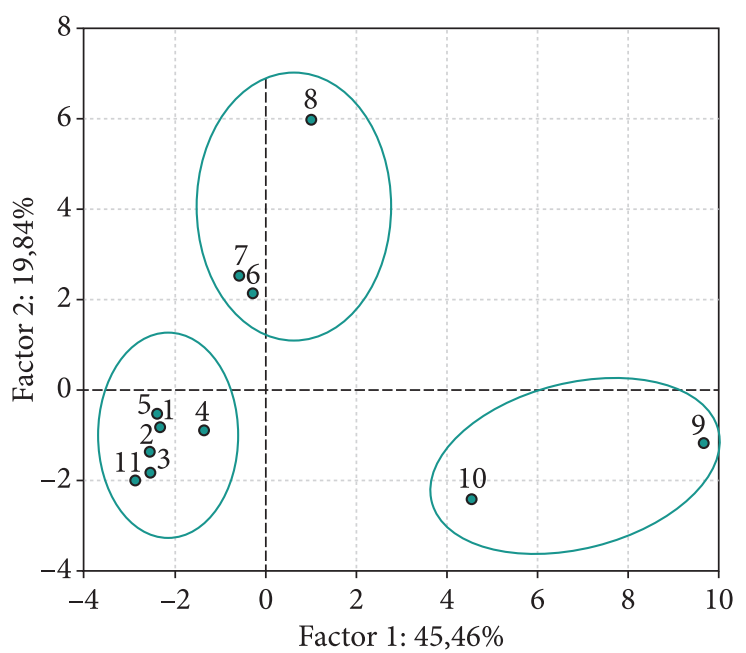

Figura 14 Análisis de componentes principales (PCA) de los once Piscos analizados, según las similitudes composicionales de sus fracciones volátiles aisladas por HS-SPME/GC-MS (Véanse los nombres y tipo de Pisco en la Tabla 2).

espectros con los de las bases de datos o con los de compuestos estándar-, es ventajoso y mucho más eficiente que el de otras técnicas analíticas.

El resultado del análisis por GC-MS de un extracto de una tableta, una cápsula o una pastilla de la presunta droga sicotrópica, permite esclarecer varios aspectos, entre ellos: 1) cuáles son los componentes principales (principios activos) y en qué cantidad se encuentran (se requieren patrones certificados y la debida calibración; en GC-MS ello generalmente se hace en el modo de monitoreo de ion(es) seleccionado(s), SIM); 2) cuáles son las impurezas, adulterantes, precursores o productos intermedios de la síntesis presentes generalmente a nivel de trazas, en la mezcla; 3) qué solventes residuales $u$ otros componentes volátiles, productos de una contaminación cruzada o, al contrario, enmascaradores de olores, se encuentran en la mezcla. El análisis de todos estos componentes permite hacer la llamada perfilación de la droga ilícita, que es como su "huella digital" o firma (signature investigation). Ello, no solamente permite identificarla y caracterizarla, 
TIC: 47115AM1.D

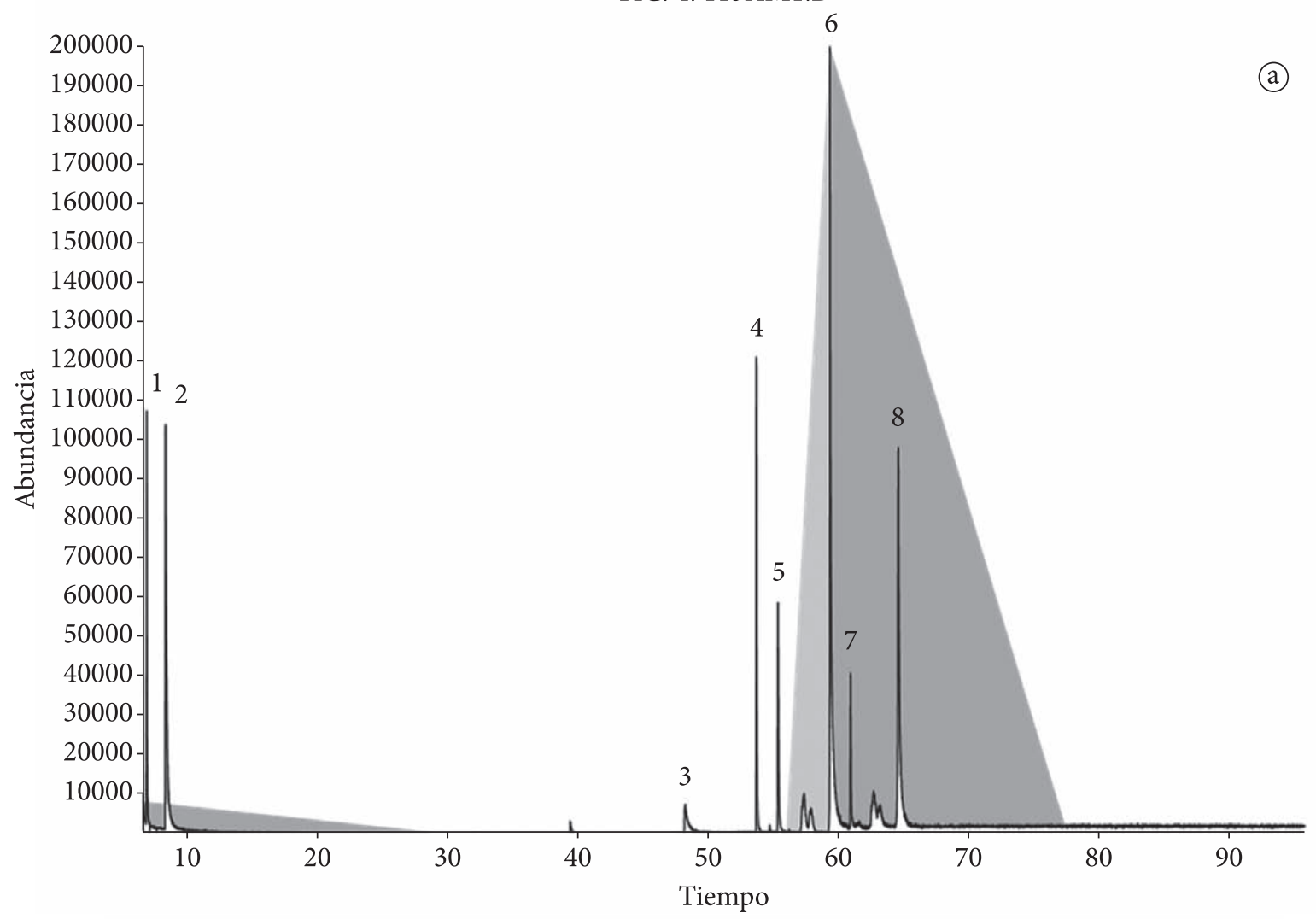

\section{Preparación de la muestra}

- Pesaje, homogeneización, cuarteo y toma de una alícuota

- Extracción sólido-líquido

$\left(\mathrm{CH}_{2} \mathrm{Cl}_{2}\right.$ : isopropanol $\left.85: 15 ; 1 \mathrm{~mL} \times 3\right)$

- Concentración del extrato $(1 \mathrm{~mL})$

- Análisis instrumental: GC-MS, inyección splitless, coluna DB-5 (60 m)

\section{Identificación}

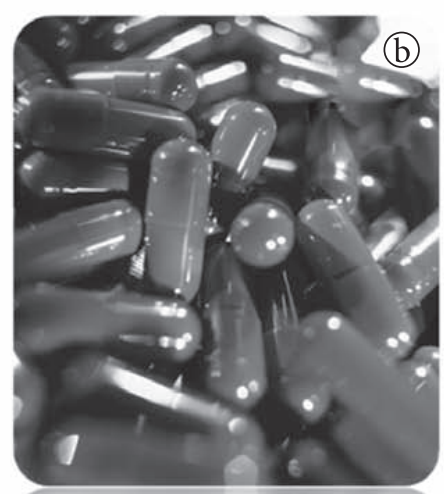

1. Ciclohexeno

2. 1-Bromo-butano

3. Cafeína

4. Bromuro de metil-escopolamina

5. Derivado de escopolamina

6. Escopolamina

7. Compuesto nitrogenado (N.I.)

7. Acetato de escopolamina

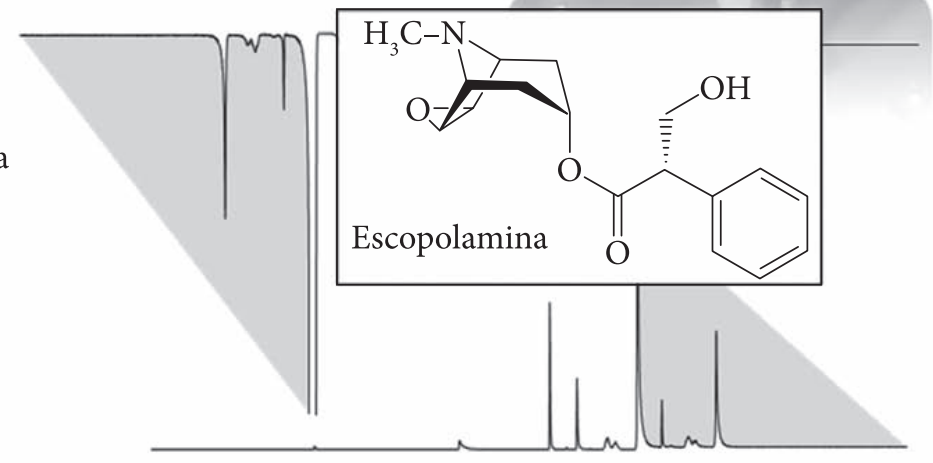

Figura 15 a) Cromatograma típico del extracto obtenido de una pastilla de droga de diseño (droga de síntesis, street drug). GC-MS (El, 70 eV). Columna DB-5 $(60 \mathrm{~m})$. b) Modo de preparación del extracto y su composición química: escopolamina - compuesto mayoritario. 
sino relacionarla con su origen (modo de síntesis, reactivos y solventes empleados, precursores, etc.) y, en algunos casos, con su fabricante (país, ciudad). Frecuentemente se logra establecer el vínculo entre los distribuidores quienes realizan la dilución o adulteración (cutting) del producto, para su venta al por menor. En la Figura 15 aparece la corriente iónica total (GC-MS, EI, 70 eV) del extracto de una pastilla decomisada en una discoteca; ésta fue vendida como una pastilla de "Extacy" (MDMA, 3,4-metilenodioximetilanfetamina), pero, en realidad, resultó ser una peligrosa mezcla que contenía cafeína, escopolamina (alcaloide tropánico, presente en varias plantas de la familia Solanácea, por ejemplo en géneros Datura o Brugmansia) y sus derivados.

\section{Referencias}

1 García C, Herrero S, Pérez JL, Moreno B. A simplified Quick, Easy, Cheap, Effective, Rugged and Safe approach for the determination of trihalomethanes and benzene, toluene, ethylbenzene and xylenes in soil matrices by fast gas chromatography with mass spectrometry detection. Analytica Chimica Acta 2011; 689(1):129-136. PMid:21338768. http://dx.doi. org/10.1016/j.aca.2011.01.023

2 Pérez JL, Herrero S, García C, Moreno B. Programmed temperature vaporizer based method for the sensitive determination of trihalomethanes and benzene, toluene, ethylbenzene and xylenes in soils. Journal of Chromatography A 2009; 1216(32):60636070. PMid:19577756. http://dx.doi.org/10.1016/j. chroma.2009.06.057

3 Pérez JL, Herrero S, García C, Moreno B. Headspaceprogrammed temperature vaporizer-fast gas chromatography-mass spectrometry coupling for the determination of trihalomethanes in water. Journal of Chromatography A 2008; 1194(1):103110. PMid:18482730. http://dx.doi.org/10.1016/j. chroma.2008.04.037

4 Zhang Z, Li G. A review of advances and new developments in the analysis of biological volatile organic compounds. Microchemical Journal 2010; 95(2):127-139. http://dx.doi.org/10.1016/j. microc.2009.12.017
$4 \quad$ Wang L, Li M, Jin W, Li S, Zhang S, Yu L. Variations in the components of Osmanthus fragrans Lour. essential oil at different stages of flowering. Food Chemistry 2009; 114(1):233-236. http://dx.doi.org/10.1016/j. foodchem.2008.09.044

6 Chalchat J-C, Özcan MM. Comparative essential oil composition of flowers, leaves and stems of basil (Ocimum basilicum L.) used as herb. Food Chemistry 2008; 110(2):501-503. http://dx.doi.org/10.1016/j. foodchem.2008.02.018

7 Stashenko E, Ordóñez SA, Marín NA, Martínez JR. Determination of the volatile and semi-volatile secondary metabolites, and aristolochic acids in Aristolochia ringens Vahl. Journal of Chromatographic Science, 2009; 47(9):817-821.

8 Pedroza MA, Zalacain A, Lara JF, Salinas MR. Global grape aroma potential and its individual analysis by SBSE-GC-MS. Food Research International 2010; 43(4):1003-1008. http://dx.doi.org/10.1016/j. foodres.2010.01.008

9 Courtois EA, Paine CET, Blandinieres P-A, Stien D, Bessiere JM, Houel E et al. Diversity of the volatile organic compounds emitted by 55 species of tropical trees: a survey in French Guiana. Journal of Chemical Ecology 2009; 35(11):1349-1362. PMid:20012675. http://dx.doi.org/10.1007/s10886-009-9718-1

10 Goodwin TE, Brown PA, Eggert MS, Evola MG, House SJ, Morshedi RG et al. Use of automated solid phase dynamic extraction (SPDE)/GC-MS and novel macros in the search for african elephant pheromones. Chemical Signals in Vertebrates 2008;11(I):25-35. http://dx.doi.org/10.1007/978-0-387-73945-8_2

11 Riu-Aumatell M, Vargas L, Vichi S, Guadayol JM, López-Tamames E, Buxaderas S. Characterisation of volatile composition of white salsify (Tragopogon porrifolius L.) by headspace solid-phase microextraction (HS-SPME) and simultaneous distillation-extraction (SDE) coupled to GC-MS. Food Chemistry 2011; 129(2):557-564.

12 Sandercock PML. Fire investigation and ignitable liquid residue analysis - A review: 2001-2007. Forensic Science International 2008;176(2-3):93110. PMid:17949931. http://dx.doi.org/10.1016/j. forsciint.2007.09.004

13 Monfreda M, Gregori A. Differentiation of unevaporated gasoline samples according to their brands, by SPME-GC-MS and multivariate statistical analysis. Journal of Forensic Sciences 2011; 56(2):372-380. PMid:21265832. http://dx.doi. org/10.1111/j.1556-4029.2010.01644.x 
14 Yoshida H, Kaneko T, Suzuki S. A solid-phase microextraction method for the detection of ignitable liquids in fire debris. Journal of Forensic Sciences 2008; 53(3):668-676. PMid:18471212. http://dx.doi. org/10.1111/j.1556-4029.2008.00704.x

15 McCord B, Bottegal M, Mathis J. Mass spectrometry for the analysis of low explosives. Detection of biological agents for the prevention of bioterrorism. NATO Science for Peace and Security Series A: Chemistry and Biology 2011; 221-229. http://dx.doi. org/10.1007/978-90-481-9815-3_14

16 Dalby O, Butler D, Birkett JW. Analysis of gunshot residue and associated materials - A review. Journal of Forensic Sciences 2010; 55(4):924-943. PMid:20384934. http://dx.doi.org/10.1111/j.1556-4029.2010.01370.x

17 Hoffman EM, Curran AM, Dulgerian N, Stockham RA, Eckenrode BA. Characterization of the volatile organic compounds present in the headspace of decomposing human remains. Forensic Science International 2009; 186(1-3):6-13. PMid:19203852. http://dx.doi.org/10.1016/j.forsciint.2008.12.022

18 Castiglioni S, Zuccato E, Chiabrando C, Fanelli R, Bagnati R. Mass spectrometric analysis of illicit drugs in wastewater and surface water. Mass Spectrometry Reviews, 2008; 27(4):378-394. PMid:18421768. http:// dx.doi.org/10.1002/mas.20168

19 Turner C. Potential of breath and skin analysis for monitoring blood glucose concentration in diabetes. Expert Review of Molecular Diagnostics 2011; 11(5):497-503. PMid:21707458. http://dx.doi. org/10.1586/erm.11.31

20 Song G, Qin T, Liu H, Xu GB, Pan Y-Y, Xiong F-X et al. Quantitative breath analysis of volatile organic compounds of lung cancer patients. Lung Cancer 2010; 67(2):227-231. PMid:19409642. http://dx.doi. org/10.1016/j.lungcan.2009.03.029

21 Mebazaa R, Rega B, Camel V. Analysis of human male armpit sweat after fenugreek ingestion: Characterisation of odour active compounds by gas chromatography coupled to mass spectrometry and olfactometry. Food Chemistry 2011; 128(1):227-235. http://dx.doi.org/10.1016/j.foodchem.2011.02.063

22 Soini HA, Klouckova I, Wiesler D, Oberzaucher E, Grammer K, Dixon SJ et al. Analysis of volatile organic compounds in human saliva by a static sorptive extraction method and gas chromatographymass spectrometry. Journal of Chemical Ecology 2010; 36(9):1035-1042. PMid:20809147. http://dx.doi. org/10.1007/s10886-010-9846-7
23 Probert CSJ, Ahmed I, Khalid T, Johnson E, Smith S, Ratcliffe N. Volatile organic compounds as diagnostic biomarkers in gastrointestinal and liver diseases. Journal of Gastrointestinal and Liver Disease 2009;18(3):337-343. PMid:19795029.

24 Kataoka H, Saito K. Recent advances in SPME techniques in biomedical analysis. Journal of Pharmaceutical and Biomedical Analysis 2011; 54(5):926-950. PMid:21194868. http://dx.doi. org/10.1016/j.jpba.2010.12.010

25 Pasikanti KK, Ho PC, Chan ECY. Gas chromatography/ mass spectrometry in metabolic profiling of biological fluids. Journal of Chromatography B 2008; 871(2):202211. PMid:18479983. http://dx.doi.org/10.1016/j. jchromb.2008.04.033

26 Logue BA, Hinkens DM, Baskin SI, Rockwood GA. The analysis of cyanide and its breakdown products in biological samples. Critical Reviews in Analytical Chemistry 2010; 40(2):122-147. http://dx.doi. org/10.1080/10408340903535315

27 LeDoux M. Analytical methods applied to the determination of pesticide residues in foods of animal origin. A review of the past two decades. Journal of Chromatography A 2011; 1218(8):10211036. PMid:21236435. http://dx.doi.org/10.1016/j. chroma.2010.12.097

28 Marriott PJ, Eyres GT, Dufour JP. Emerging opportunities for flavor analysis through hyphenated gas chromatography. Journal of Agricultural and Food Chemistry 2009; 57:9962-9971. PMid:19817416. http://dx.doi.org/10.1021/jf9013845

29 López-Feria S, Cárdenas S, Valcárcel M. Simplifying chromatographic analysis of the volatile fraction of foods. TrAC Trends in Analytical Chemistry 2008; 27(9):794-803. http://dx.doi.org/10.1016/j. trac.2008.07.006

30 Barata A, Campo E, Malfeito-Ferreira M, Loureiro V, Cacho J, Ferreira V. Analytical and sensorial characterization of the aroma of wines produced with sour rotten grapes using GC-O and GC-MS: Identification of key aroma compounds. Journal of. Agricultural and Food Chemistry 2011; 59:2543-2553. PMid:21348497. http://dx.doi.org/10.1021/jf104141f

31 Ikem A. Measurement of volatile organic compounds in bottled and tap waters by purge and trap GC-MS: Are drinking water types different? Journal of Food Composition and Analysis 2010; 23(1):70-77. http:// dx.doi.org/10.1016/j.jfca.2009.05.005 
32 Zellner B, Dugo P, Dugo G, Mondello L. Gas chromatography-olfactometry in food flavour analysis. Journal of Chromatography A 2008; 1186(1-2):123143. http://dx.doi.org/10.1016/j.chroma.2007.09.006

33 Linsinger TPJ, Birgersson-Liebich A, Lamberty A, Pellizzato F, Venelinov T, Voorspoels S. Development of the first certified reference materials for several brominated flame retardants in polymers. Analytical. Chemistry 2009; 81:3792-3800. http://dx.doi. org/10.1021/ac900139r

34 Kawakami T, Isama K, Matsuoka A. Analysis of phthalic acid diesters, monoester, and other plasticizers in polyvinyl chloride household products in Japan. Journal of Environmental Science and Health, Part A 2011; 46(8):855-864.

35 Odabasi M. Halogenated volatile organic compounds from the use of chlorine-bleach-containing household products. Environmental Science and Technology 2008; 42(5):1445-1451. http://dx.doi.org/10.1021/ es702355u

36 Salgueiro L, Martins AP, Correia H. Raw materials: the importance of quality and safety. A review. Flavour and Fragrance Journal 2010; 25(5):253-271. http:// dx.doi.org/10.1002/ffj.1973

37 Nagpal S, Karan, Upadhyay A, Bhardwaj R, Thakkar A. A review on need and importance of impurity profiling. Current Pharmaceutical Analysis 2011; 7(1):62-70. http://dx.doi.org/10.2174/157341211794708749

38 Risby TH, Solga SF. Current status of clinical breath analysis. Applied Physics B: Lasers and Optics 2006; 85(2-3):421-426. http://dx.doi.org/10.1007/s00340006-2280-4

39 Stashenko E, Martinez J. Sampling flower scent for chromatographic analysis. Journal of Separation Science 2008; 31(11):2022-2031. http://dx.doi. org/10.1002/jssc.200800151

40 Korhoňová M, Hron K, Klimčíková D, Müller L, Bednář P, Barták P. Coffee aroma-Statistical analysis of compositional data. Talanta 2009; 80(2):710-715. http://dx.doi.org/10.1016/j.talanta.2009.07.054

41 Król S, Zabiegała B, Namieśnik J. Monitoring VOCs in atmospheric air II. Sample collection and preparation. TrAC Trends in Analytical Chemistry 2010; 29,(9):1101-1112. http://dx.doi.org/10.1016/j. trac.2010.05.010

42 Van Dam NM, Poppy GM. Why plant volatile analysis needs bioinformatics - detecting signal from noise in increasingly complex profiles. Plant Biology 2008; 10(1):29-37. http://dx.doi.org/10.1055/s-2007-964961
43 Dincer F, Odabasi M, Muezzinoglu A. Chemical characterization of odorous gases at a landfill site by gas chromatography-mass spectrometry. Journal of Chromatography A 2006; 1122(1-2):222-229. http:// dx.doi.org/10.1016/j.chroma.2006.04.075

44 Nijssen LM, Visscher CA, Maarse H, Wilemsens LC, Boelens MH. Volatile Compounds in Food. Qualitative and Quantitative Data. 7rd ed. Zeist, The Netherlands: TNO Nutrition and Food Research Institute; 1996.

45 Woolfenden E. Sorbent-based sampling methods for volatile and semi-volatile organic compounds in air: Part 1: Sorbent-based air monitoring options. Journal of Chromatography A 2010; 1217(16):2674-2684. http://dx.doi.org/10.1016/j.chroma.2009.12.042

46 Bohlin P, Jones KC, Strandberg B. Occupational and indoor air exposure to persistent organic pollutants: A review of passive sampling techniques and needs. Journal of Environmental Monioring 2007; 9:501-509. http://dx.doi.org/10.1039/b700627f

47 Stashenko E, Wong JW, Martínez JR, Mateus A, Shibamoto T. High resolution gas chromatography nitrogen - phosphorous detection of saturated volatile aldehydes derivatized with 2-hydrazinobenzothiazole. Journal of Chromatography A 1996; 752:209. http:// dx.doi.org/10.1016/S0021-9673(96)00494-3

48 Stashenko E, Puertas MA, Salgar W, Delgado W, Martínez J. Solid-phase microextraction with on-fibre derivatisation applied to the analysis of volatile carbonyl compounds. Journal of Chromatography A 2000; 886:175181. http://dx.doi.org/10.1016/S0021-9673(00)00479-9

49 Stashenko E, Macku C, Shibamoto T. Monitoring volatile chemicals formed from must during yeast fermentation. Journal of Agricultural and Food Chemistry 1992; 40(11):2257-2259. http://dx.doi. org/10.1021/jf00023a040

50 Stashenko E, Martínez JR. Algunos aspectos prácticos para la identificación de analitos por cromatografía de gases acoplada a espectrometría de masas. Scientia Chromatographica 2010; 2 (1):29-47.

51 Stashenko E, Martínez JR. Separar, fragmentar e integrar: la rutina de un análisis por GC-MS. Patrones de fragmentación de moléculas orgánicas. Scientia Chromatographica 2010; 2(2):23-46.

52 Stashenko E, Martínez JR. GC-MS: Más de un analizador de masas, ¿para qué?. Scientia Chromatographica 2010; 2(4):33-59.

Recebido: 28/07/2011 Aceito: 03/08/2011 
Anexo 1 Abreviaturas.

\begin{tabular}{|c|c|}
\hline BTEX & benceno, tolveno, etilbenceno y xilenos \\
\hline $\mathrm{Cl}$ & ionización química \\
\hline D.I. & diámetro interno \\
\hline$d_{f}$ & grosor de la fase estacionaria. \\
\hline ECD & detector de captura electrónica \\
\hline $\mathrm{El}$ & ionización por impacto electrónico \\
\hline FID & detector de ionización en llama \\
\hline FPD & detector fotométrico de llama \\
\hline FT-ICR-MS & espectrometría de masas de resonancia ion-ciclotrón con transformada de Fourier \\
\hline GC & cromatografía de gases \\
\hline GC-MS & cromatografía de gases acoplada a espectrometría de masas \\
\hline HD & hidrodestilación \\
\hline HS & espacio de cabeza, fase vapor \\
\hline LVI & inyección de volúmenes grandes de muestra \\
\hline MS & espectrometría de masas \\
\hline MSD & detector selectivo de masas \\
\hline MWAE & extracción asistida por la radiación de microondas \\
\hline $\mathrm{NICl}$ & ionización química de iones negativos \\
\hline P\&T & purga y trampa \\
\hline PDMS/DVB & polidimetilsiloxano/divinilbenceno \\
\hline PFPH & pentafluorofenilhidracina \\
\hline $\mathrm{PI} \mathrm{Cl}$ & ionización química con iones positivas \\
\hline PTV & inyector de temperatura programada \\
\hline SBSE & extracción por sorción en barra de agitación \\
\hline SD & destilación por arrastre con vapor \\
\hline SDE & destilación-extracción simultánea con solvente \\
\hline SFE & extracción con fluido supercrítico \\
\hline SIM & monitoreo de iones selectivos \\
\hline SPE & extracción en fase sólida \\
\hline SPME & microextracción en fase sólida \\
\hline STE & extracción por sorción sobre cinta sorbente \\
\hline TCD & detector de conductividad térmica \\
\hline TD & desorción térmica \\
\hline THM & trihalometanos \\
\hline $\mathrm{TIC}$ & corriente iónica total \\
\hline TOF & analizador de masas de tiempo de vuelo \\
\hline VOC & compuestos orgánicos volátiles \\
\hline
\end{tabular}

